\title{
A Recognition Methodology for the Key Geometric Errors of a Multi-Axis Machine Tool Based on Accuracy Retentivity Analysis
}

\author{
Shijie Guo $\mathbb{i})^{1,2}$ Shufeng Tang, ${ }^{1}$ and Dongsheng Zhang $\mathbb{D}^{2}$ \\ ${ }^{1}$ The College of Mechanical Engineering, Inner Mongolia University of Technology, Hohhot 010051, China \\ ${ }^{2}$ School of Mechanical Engineering, State Key Laboratory for Manufacturing Systems Engineering, Xi'an Jiaotong University, \\ Xi'an 710049, China \\ Correspondence should be addressed to Dongsheng Zhang; zdsxjtul1974@gmail.com
}

Received 4 May 2019; Revised 11 August 2019; Accepted 28 August 2019; Published 22 November 2019

Academic Editor: Qingling Wang

Copyright (C) 2019 Shijie Guo et al. This is an open access article distributed under the Creative Commons Attribution License, which permits unrestricted use, distribution, and reproduction in any medium, provided the original work is properly cited.

\begin{abstract}
This paper proposes a recognition methodology for key geometric errors using the feature extraction method and accuracy retentivity analysis and presents the approach of optimization compensation of the geometric error of a multiaxis machine tool. The universal kinematics relations of the multiaxis machine tool are first modelled mathematically based on screw theory. Then, the retentivity of geometric accuracy with respect to the geometric error is defined based on the mapping between the constitutive geometric errors and the time domain. The results show that the variation in the spatial error vector is nonlinear while considering the operation time of the machine tool and the position of the motion axes. Based on this aspect, key factors are extracted that simultaneously consider the correlation, similarity, and sensitivity of the geometric error terms, and the results reveal that the effect of the position-independent geometric errors (PIGEs) on the error vectors of the position and orientation is greater than that of the position-dependent geometric errors (PDGEs) of the linear and rotary axes. Then, the fruit fly optimization algorithm (FOA) is adopted to determine the compensation values through multiobjective tradeoffs between accuracy retentivity and fluctuation in the geometric errors. Finally, an experiment on a four-axis horizontal boring machine tool is used to validate the effectiveness of the proposed approach. The experimental results show that the variations in the precision of each test piece are lower than $25.0 \%$, and the maximum variance in the detection indexes between the finished test pieces is $0.002 \mathrm{~mm}$ when the optimized parameters are used for error compensation. This method not only recognizes the key geometric errors but also compensates for the geometric error of the machine tool based on the accuracy retentivity analysis results. The results show that the proposed methodology can effectively enhance the machining accuracy.
\end{abstract}

\section{Introduction}

1.1. Literature Survey. For a precision CNC machine tool, the quasistatic error, which consists of the geometric error and thermal error, accounts for approximately $70 \%$ of the total error [1]. In modern manufacturing, controlling the temperature of the thermally sensitive components and environment can effectively eliminate the influence of thermally induced error. Under this condition, the geometric error accounts for $40 \%$ of the total error [2]. The analysis of the geometric error and volumetric error compensation method of multiaxis CNC machine tools has become a key factor in improving their precision.
The accuracy of a machine tool inevitably deteriorates due to the effect of error sources over extended tool operation [3]. The fundamental cause for these problems lies in the design tolerance, manufacturing defects, assembly error arising from the manufacturing process, and wear occurring during the operation of machine the tool. The precision of the machine tool can be impacted by the two factor types (geometric and thermal) mentioned above. For instance, the positioning accuracy of the linear axes is related to the mounting precision of the joint surface; moreover, slideguide wear is associated with straightness accuracy degradation of the positioning accuracy. The geometric error provides a direct reflection of accuracy for a machine tool 
[4]. Based on the state of measurement and identification methods and compensation technology of geometric errors, maintaining and improving geometric accuracy retentivity during the operation of machine tools is emerging as the foremost concern for the users, and the crucial problems need to be solved first, one of which is the mapping of geometric error terms and accuracy retentivity in the time domain. Another problem is to improve the geometric accuracy based on evaluations of key geometric errors.

With a focus on these challenges, the concept and related technologies of error avoidance and error compensation are put forward. Geometric error modelling is a key part of error compensation technology and also for the basis for decoupling identification [5, 6]. Many beneficial explorations and studies on high-quality modelling have been conducted by researchers, and some effective methods have been proposed and applied to modelling, such as the product of exponentials [7, 8], homogeneous transformation matrices (HTMs) method based on multibody system theory [9], differential transformation method $[10,11]$, screw theory method $[6,12,13]$, and parametric polynomial method [14]. Fu et al. [15] established the rotation twist and rotation product of exponential formulas of rotary axes with a clear geometric meaning of twists to describe their positions and motions, and the corresponding POE formulas of squareness errors were established by analysing their geometric definition. Lee et al. [16] established a representation of position-dependent geometric errors as higher-order polynomials with $C^{1}$ continuity. Du et al. [17] established a geometric error model for the linear axis based on the Jacobian-torsor theory, and the key error sources were extracted by conducting a quantitative sensitivity analysis. Tang et al. [18] proposed a geometric error modelling approach for a six-axis stage based on the stream of variation, revealing that errors propagate and deviations accumulate on the linear axis. Fan et al. [19] adopted a truncated Fourier series function to express the curve of a guideway surface; the mapping between the tolerance and geometric errors of the guideways of machine tools was formulated; and mapping relations between error vectors and geometric error terms were established.

The analysis of geometric error terms has been an active research topic in the past 3 years due to the implementation of both offline error compensation and real-time error compensation $[6,20]$. The fundamental reason is that the precision of the machine tool can be further enhanced by analysing the correlation effect and nonlinear characteristics of geometric error terms [21, 22]. Evaluation or analysis of geometric error sources is fundamentally important, whether for error compensation or precision design for improving machine tool accuracy retentivity [23]. Data-based sensitivity has been applied in a large spectrum of domains and problems $[24,25]$, and sensitivity analysis can be considered a wrapper method, according to the categorization identified in references [26, 27]. Sensitivity analysis may also be included within the model training process of embedded feature extraction [28]. The concept of accuracy retentivity of the machine tool was quantitatively defined by Wang et al. [29], and an analysis model of the spatial position accuracy of a three-axis machine tool based on multibody theory was established. According to the definition of accuracy retentivity of CNC machine tools, the accuracy retentivity of machine tools is evaluated in the time domain when the accuracy indexes of machine tools are kept within the required range $[29,30]$. The longer the time is, the better the accuracy retentivity of the machine tool is, and the opposite case also holds. However, aiming at the problems of the accuracy variations of machine tools, most studies have focused on the accuracy degradation and accuracy reliability evaluation caused by the wearing of guideways, bolt relaxation, and stress relaxation-induced deformation of machine bodies $[29,31-33]$. It is obvious that all of these factors reduce the geometric accuracy gradually, which affects the geometric accuracy retentivity in an essential way [34]. The main difference between accuracy reliability and accuracy retentivity is that the reliability of $\mathrm{CNC}$ machine tools is generally characterized by the fault possibility of functional components, while accuracy retentivity research mainly studies the accuracy variations of a machine tool in the time domain [29, 30]. The performance degradation of functional components is characterized by high nonlinearity, long periods, and various modes and is influenced by the processing method, lubrication method, pretightening force, and installation type. Therefore, it is very difficult to obtain performance degradation samples over the whole life cycle [32]; in this sense, machine accuracy and precision reliability assessment rely more on the accuracy of the reliability model of the machine tool. In fact, the variations of the geometric accuracy of machine tools arise from the macroscopic state of degradation of the functional components $[31,34]$, corresponding to the comprehensive embodiment of machine tool wear, manufacturing defects, and assembly deviation of the components. Geometric accuracy is measurable with special measuring instruments and methods. To our knowledge, there are no relevant evaluation criteria or effective methods for geometric accuracy retentivity of machine tools except for the definition of geometric accuracy retentivity. Therefore, research on the accuracy retentivity of machine tools has received great attention.

The four-axis horizontal boring machine, which is the key equipment for processing box components, is a specialized but common type of multiaxis machine tool. However, geometric error modelling has unique characteristics both in quantity and in the intrinsic nature of the geometric error terms for different types of machine tools $[35,36]$. For example, there are a total of 30 geometric error terms for a four-axis horizontal boring machine, comprising 24 PDGEs and 6 PIGEs [37]; for a five-axis machine tool with a tilting rotary table, there are 30 PDGEs and 11 PIGEs, and a five-axis machine tool with a tilting head and rotary table has 30 PDGEs and 13 PIGEs $[38,39]$. This means that the well-developed geometric error models for the threeand five-axis machine tools cannot describe the quantitative relations between the errors and error vectors directly for the four-axis machine tool. 
1.2. Motivations and Potential Applications. The method of calibrating and identifying geometric errors on multiaxis machine tools has been specified by the ISO-230 series through researcher effort. However, previous conclusions and methods will be further improved if the following important aspects are fully considered. (1) The existing literature on geometric error modelling focuses mainly on three- and five-axis machine tools by using multibody system theory and homogeneous coordinate transformation methods. PIGEs are treated as functions of angular errors and the position of the corresponding rotary axis [40], which is inconsistent with the geometric definition of PIGEs. Nevertheless, the configuration of a fouraxis horizontal boring machine with a turntable has received little attention, and there are few studies on geometric error modelling for four-axis machine tools in as few local coordinates as possible [37]. (2) The key geometric errors are identified by conducting a variance-based sensitivity analysis [38]; however, the variance is only a probabilistic statistical feature of geometric error terms as random variables and is inadequate to describe the uncertainty of a model input and output based only on variance-based analysis in the case of multidimensional output. Previous studies have focused mainly on geometric error analysis based on the method of variance analysis from the perspective of the precision design of machine tools $[21,23,38]$; more research on the quantitative relationship and the degree of relative importance of geometric error for determining the key geometric error terms is needed. (3) The results of sensitivity analysis and accuracy retentivity analysis are rarely directly applied in error compensation of machine tools from the view of improving compensation accuracy [39].

This work proposes a recognition methodology for key geometric errors using a feature extraction method and accuracy retentivity analysis and presents the approach of optimization compensation of multiaxis machine tools. The contributions are listed as follows. (1) The forward kinematics of the four-axis horizontal boring machine are modelled based on screw theory, and the 24 PDGEs and 6 PIGEs particular to four-axis horizontal boring machine are represented with error motion twists. The established geometric error model describes the relative motion between components of the machine tool without establishing the local coordinate on each of the moving parts as the modelling method with HTMs. (2) A new method to analyse the retentivity of geometric accuracy with respect to the geometric error is proposed based on the mapping between geometric errors and the time domain. The evaluation criterion is established based on the feature selection method, which integrates the correlation, similarity, and sensitivity of geometric error terms, and a filtering algorithm for recognizing key geometric error terms is proposed to establish the set of key geometric error elements. (3) Error compensation based on the results of the recognition methodology for the key geometric errors is proposed to improve the geometric accuracy of the fouraxis horizontal boring machine, and the main idea of key geometric error recognition and compensation methodology can also be applied to multiaxis machine tools with other configurations.

1.3. Structure of the Paper. This paper mainly aims to propose a new methodology to guarantee the retentivity of geometric accuracy and recognize key errors of multiaxis machine tool. The methodology presented in this paper, consisting of three main steps, is summarized in Figure 1.

The structure of the paper is as follows: In Section 2, geometric error modelling of the multiaxis machine tool is presented under global coordinate systems. In Section 3, the accuracy retentivity model of the geometric error for the multiaxis machine tool is established, and the recognition process of key factors based on the feature extraction method is described in detail. In Section 4, experiments are carried out on a four-axis machine tool to validate the effectiveness of the analysis method. Finally, some conclusions are drawn.

\section{Geometric Error Modelling of a Multiaxis Machine Tool}

Multiaxis machine tools contain two kinematic chains: the workpiece kinematic chain of the motion axes consists of a reference coordinate system, linear axes, and a rotary table. Similarly, the tool kinematic chain of the motion axes consists of a reference coordinate system, linear axes, and a cutting tool. The whole kinematic chain from the workpiece to the cutting tool is composed of the above two open-loop kinematic chains. The relative motion between the cutting tool and the workpiece of the end of the two kinematic chains generates the cutter path.

2.1. Geometric Error Modelling for the Four-Axis Machine Tool. In this paper, the displacement of all axes in the initial position at time zero is defined as the initial state of the machine tool structure. According to screw theory [41], the homogeneous transformation matrix of the $n$-th axis relative to the reference coordinate system is

$$
\left[g_{b n}\left(\theta_{1}, \theta_{2}, \ldots, \theta_{n}\right)\right]=e^{\widehat{\xi}_{1} \theta_{1}} e^{\widehat{\xi}_{2} \theta_{2}}, \ldots, e^{\widehat{\xi}_{n} \theta_{n}}\left[g_{b n}(0)\right],
$$

where $\left[g_{b n}(0)\right]$ is the homogeneous transformation matrix of the $n$-th axis in the reference structure relative to the reference coordinate system. In equation $(1), \xi_{i}(1,2, \ldots, n)$ and $\theta_{i}(1,2, \ldots, n)$ represent the screw and displacement of the $i$-th axis relative to the reference coordinate system, respectively.

For the rotary axis of the multiaxis machine tool, the screw of the revolute joint is calculated based on screw theory as follows:

$$
\begin{aligned}
\xi_{i} & =\left[\begin{array}{c}
v_{i} \\
\omega_{i}
\end{array}\right], \\
v_{i} & =-\omega_{i} \times q_{i},
\end{aligned}
$$

where $\omega_{i} \in R^{3}$ represents the unit direction vector of the rotary axis and $q_{i} \in R^{3}$ is the arbitrary points on the rotary axis, which can be represented in the machine tool 


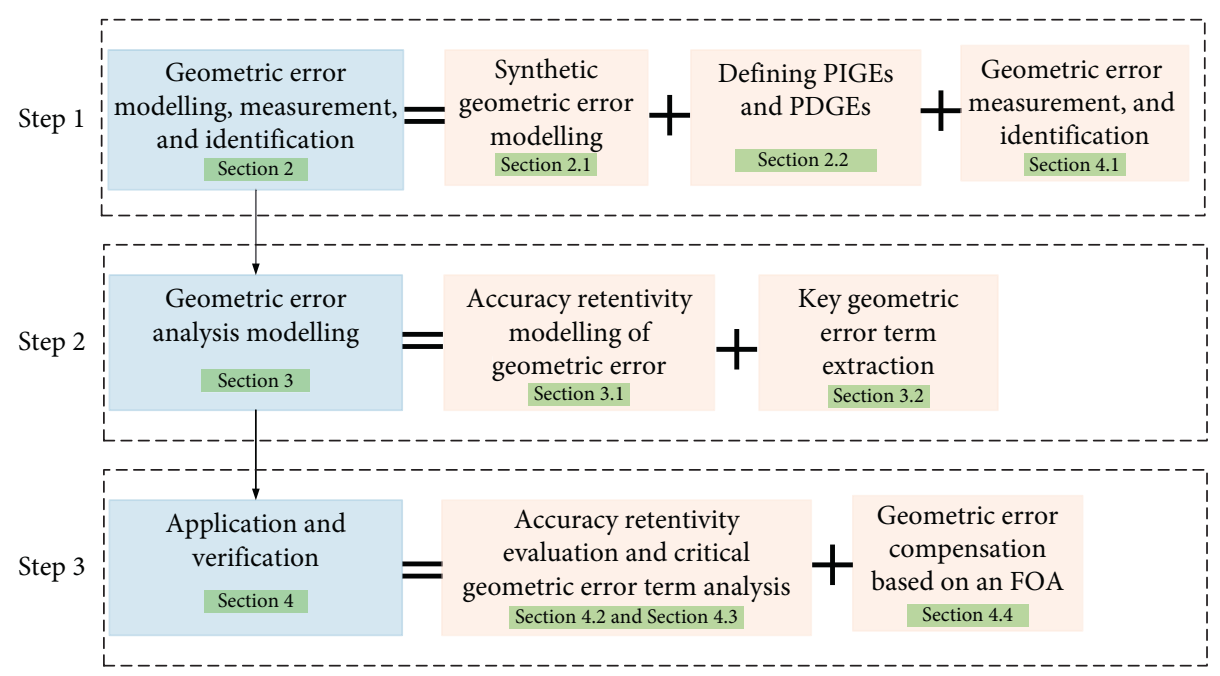

FIGURE 1: Framework of the proposed methodology.

coordinate system, i.e., for the $A-, B$-, and $C$-axes, $\omega_{a}=$ $\left[\begin{array}{lll}1 & 0 & 0\end{array}\right]^{\mathrm{T}}, \quad \omega_{b}=\left[\begin{array}{lll}0 & 1 & 0\end{array}\right]^{\mathrm{T}}$, and $\omega_{c}=\left[\begin{array}{lll}0 & 0 & 1\end{array}\right]^{\mathrm{T}}, \quad$ respectively, and $q=\left[\begin{array}{lll}0 & 0 & 0\end{array}\right]^{\mathrm{T}}$, where $q$ is defined as coincidence with the machine tool coordinate system; finally, " $x$ " represents cross multiplication. Note that vector $\omega$ is expressed and correlated in the matrix form at this time. The exponential expression of twist is

$$
e^{\widehat{\xi}_{i} \theta_{i}}=\left[\begin{array}{ll}
e^{\widehat{\xi}_{i} \theta_{i}} & \left(\mathbf{I}_{1 \times 3}-e^{\widehat{\xi}_{i} \theta_{i}}\right)-\left(\omega_{i} \times v_{i}\right) \\
0_{1 \times 3} & 1
\end{array}\right],
$$

where

$$
e^{i} \widehat{\omega}_{i} \omega_{i}=\mathbf{I}_{3 \times 3}+\widehat{\omega}_{i} \sin \theta_{i}+\widehat{\omega}_{i}^{2}\left(1-\cos \theta_{i}\right) .
$$

$\omega_{i} \in R^{3}$ and $q_{i} \in R^{3}$ can be mapped to $\widehat{\omega}_{i}^{3} \in \operatorname{Se}(3)$ and $\widehat{q}_{i}^{3} \in \operatorname{Se}(3)$ with operator ${ }^{\wedge}$, respectively, where Se (3) and So (3) are sets of $3 \times 3$ matrices that satisfy the special orthogonal with respect to $\omega_{i}$ and $q_{i}$, respectively, and operator $\checkmark$ has the opposite function compared to the former. Similarly, the screw expression of the linear axis is

$$
\begin{aligned}
\xi_{i} & =\left[\begin{array}{l}
v_{i} \\
0
\end{array}\right], \\
e^{\widehat{\xi}_{i} \theta_{i}} & =\left[\begin{array}{cc}
\mathbf{I}_{3 \times 3} & \theta_{i} v_{i} \\
0_{3 \times 3} & 1
\end{array}\right],
\end{aligned}
$$

where $v_{i} \in R^{3}$ represents the unit direction vector of the linear axis. The above derivation of the screw motion can be found in reference [41].

$\left[g_{b w}(0)\right]$ and $\left[g_{b t}(0)\right]$ are the transformation matrices of the workpiece coordinate system and tool coordinate system relative to the reference coordinate system, respectively. Here, $w_{\mathrm{o}}$ can be represented as $\left[\begin{array}{lll}w_{o x} & w_{o y} & w_{o z}\end{array}\right]^{\mathrm{T}}, t_{o}$ is equal to $\left[\begin{array}{lll}t_{o x} & t_{o y} & t_{o z}\end{array}\right]^{\mathrm{T}}$, and $\left[g_{b w}(0)\right]$ and $\left[g_{b t}(0)\right]$ can be expressed in the screw form.

$$
\begin{aligned}
\xi_{w} & =\left[\begin{array}{c}
-w_{0} \\
0_{3 \times 1}
\end{array}\right], \\
\xi_{t} & =\left[\begin{array}{c}
t_{0} \\
0_{3 \times 1}
\end{array}\right] .
\end{aligned}
$$

According to the expression of equation (1), the transformation matrix of the workpiece coordinate system relative to the reference coordinate is

$$
\left[g_{b w}\left(\theta_{1 w}, \theta_{2 w}, \ldots, \theta_{n w}\right)\right]=e^{\widehat{\xi}_{1 w} \theta_{1 w}} e^{\widehat{\xi}_{2 w} \theta_{2 w}} \ldots e^{\widehat{\xi}_{n w} \theta_{n w}}\left[g_{b w}(0)\right] .
$$

Here, $\xi_{i w}(i=1,2, \ldots, m)$ and $\theta_{i w}(i=1,2, \ldots, m)$ are, respectively, the twists and the displacements of the $i$-th moving components on the workpiece motion chain counted from the workpiece towards the reference coordinate system.

Similarly, the transformation matrix of the cutting tool coordinate system relative to the reference coordinate is

$$
\left[g_{b t}\left(\theta_{1 t}, \theta_{2 t}, \ldots, \theta_{n t}\right)\right]=e^{\widehat{\xi}_{1 t} \theta_{1 t}} e^{\widehat{\xi}_{2 t} \theta_{2 t}} \ldots e^{\widehat{\xi}_{n t} \theta_{n t}}\left[g_{b t}(0)\right] .
$$

Here, $\xi_{i t}(i=1,2, \ldots, n)$ and $\theta_{i t}(i=1,2, \ldots, n)$ are, respectively, the twists and displacements of the $i$-th moving components on the cutter kinematic chain counted from the cutting tool towards the reference coordinate system.

Combining equations (7) and (8), the homogeneous transformation matrix of the tool coordinate system relative to the workpiece coordinate system is

$$
\left[g_{w t}\left(\theta_{1 w}, \theta_{2 w}, \ldots, \theta_{n w}, \theta_{1 t}, \theta_{2 t}, \ldots, \theta_{n t}\right)\right]=\left[g_{b w}\left(\theta_{1 w}, \theta_{2 w}, \ldots, \theta_{n w}\right)\right]^{-1}\left[g_{b t}\left(\theta_{1 t}, \theta_{2 t}, \ldots, \theta_{n t}\right)\right]
$$


The equivalence relation of equation (9) is

$$
\left[g_{w b}\left(\theta_{1 w}, \theta_{2 w}, \ldots, \theta_{n w}\right)\right]=\left[g_{b w}(0)\right]^{-1} e^{-\widehat{\xi}_{1 w} \theta_{1 w}} e^{-\widehat{\xi}_{2 w} \theta_{2 w}} \ldots e^{-\widehat{\xi}_{n w} \theta_{n w}} e^{-\widehat{\xi}_{1 t} \theta_{1 t}} e^{-\widehat{\xi}_{2 t} \theta_{2 t}} \ldots e^{-\widehat{\xi}_{\xi t} \theta_{n t}}\left[g_{b t}(0)\right]
$$

The position and orientation of the tool tips are $r_{p t}$ and $r_{o t}$, respectively, and the forward kinematics for the position and orientation vectors of the cutting tool relative to the workpiece are established based on equation (1), which can be expressed as

$$
\left[\begin{array}{cc}
\mathbf{P}_{k} & \mathbf{O}_{k} \\
1 & 0
\end{array}\right]=\left[g_{w t}\left(\theta_{1 w}, \theta_{2 w}, \theta_{3 w}, \theta_{4 w},\right)\right]\left[\begin{array}{cc}
r_{p t} & r_{o t} \\
1 & 0
\end{array}\right]
$$

The abovementioned model can be directly used for calculating the position and orientation of the tool relative to the workpiece according to the displacement of each axis, without requiring establishing the local coordinate system or taking account of the relative location among the machine tool components, which is more conformable to the sole nature of the datum of measurement and assembly operation.

2.2. Machine Configuration and Definition of the Geometric Error for a Four-Axis Machine Tool. The abovementioned universal model, adapted to kinematical modelling of the four-axis machine tool depicted in Figure 2, is composed of linear axes $(X-, Y$-, and $Z$-axes) and a rotary axis ( $B$-axis) and spindle axis ( $S$-axis).

As known from the nature of rigid body motion, each motion axis has six degrees of freedom in the Cartesian coordinate system, for which the corresponding machine tool precision contains six geometric errors. The 30 geometric errors of the four-axis machine tool are listed in Table 1.

There are six PIGEs, namely, $\gamma_{x y}, \beta_{x z}, \alpha_{y z}, \alpha_{y b}, \gamma_{y b}$, and $\delta_{z b y}$ [37]; $\gamma_{x y}$ is the squareness error of the $X$-axis around the $Y$ direction, $\beta_{x z}$ is the squareness error of the $X$-axis around the $Z$ direction, $\alpha_{y z}$ is the squareness error of the $Y$-axis around the $Z$ direction, $\alpha_{y b}$ and $\gamma_{y b}$ are the angular errors of the $B$-axis with respect to the $Y$-axis about the $X$-and $Z$-axes, respectively, and $\delta_{z b y}$ is the linear shift of the $B$-axis with respect to the $Y$-axis in the $Z$ direction.

Based on the expression of the forward kinematics model (equations (9) and (10)), the position and orientation vector of the four-axis machine tool can be expressed as

$$
\left\{\begin{array}{l}
{\left[\begin{array}{llll}
\mathbf{P}_{x} & \mathbf{P}_{y} & \mathbf{P}_{z} & 1
\end{array}\right]^{\mathrm{T}}=\left[g_{b w}(0)\right]^{-1} T^{B} T^{Z} T^{X} T^{Y}\left[g_{b t}(0)\right]\left[\begin{array}{llll}
0 & 0 & 0 & 1
\end{array}\right]^{T},} \\
{\left[\begin{array}{llll}
\mathbf{O}_{x} & \mathbf{O}_{y} & \mathbf{O}_{z} & 0
\end{array}\right]^{\mathrm{T}}=\left[g_{b w}(0)\right]^{-1} T^{B} T^{Z} T^{X} T^{Y}\left[g_{b t}(0)\right]\left[\begin{array}{llll}
0 & 0 & 1 & 0
\end{array}\right]^{T} .}
\end{array}\right.
$$

Parameters $e^{\widehat{\xi}_{D i} \theta_{D i}}$ and $e^{\widehat{\xi}_{i i} \theta_{I i}}$ represent the PIGEs and PDGEs matrices of the $i$-axis, respectively. After the exponential expressions of twist are incorporated into equation (1), the transformation of these error screws of the multiaxis machine tool can be expressed in exponential matrix form as follows:

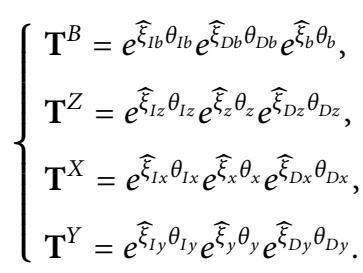

The 6 PIGEs of the four-axis machine tool listed in Table 1 can be expressed from the error twists as follows:

$$
\left\{\begin{array}{l}
e^{\widehat{\xi}_{I b} \theta_{I b}}=e^{\widehat{\xi}_{\delta z b y} \theta_{\delta z z b y}} e^{\widehat{\xi}_{x y b} \theta_{\alpha y b}} e^{\widehat{\xi}_{y y b} \theta_{y y b}}, \\
e^{\widehat{\xi}_{I z} \theta_{I z}}=e^{\widehat{\xi}_{S_{y z}} \theta_{S_{y z}} \widehat{\xi}_{\xi_{x z}} \theta_{S_{x z}}}, \\
e^{\widehat{\xi}_{I y} \theta_{I y}}=e^{\widehat{\xi}_{S_{x y}} \theta_{S_{x y}}}
\end{array}\right.
$$

Multiplication of the error motion matrix corresponding to the six PDGEs provides the motion matrix of the $i$-axis, and the PDGEs of the linear axes and rotary axis $i$ can be expressed by the products of the motion axis as follows:

$$
e^{\widehat{\xi}_{D i} \theta_{D i}}=e^{\widehat{\xi}_{\delta x i} \theta_{\delta x i}} e^{\widehat{\xi}_{\delta y i} \theta_{\delta y i}} e^{\widehat{\xi}_{\delta z i} \theta_{\delta z i}} e^{\widehat{\xi}_{s i i} \theta_{s x i}} e^{\widehat{\xi}_{s y i} \theta_{s y i}} e^{\widehat{\xi}_{s z i} \theta_{s z i}}
$$

The expressions of the transformation matrix involved in forward kinematics are as follows: 


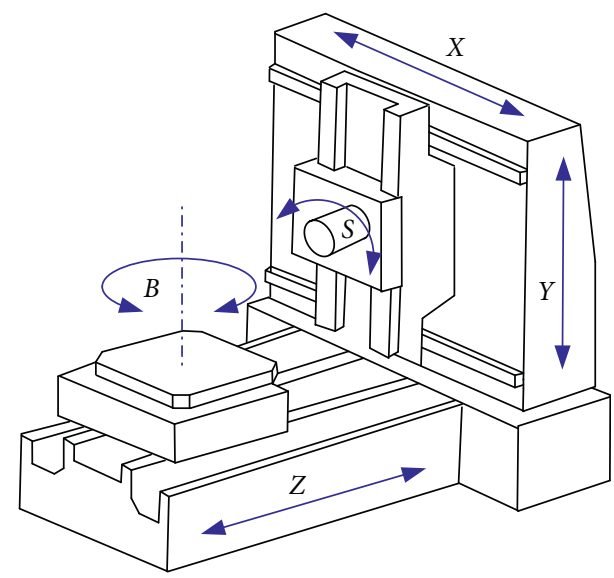

FIGURE 2: Structure schematic of the multiaxis machine tool.

TABLE 1: Geometric error terms of the four-axis machine tool.

\begin{tabular}{lcc}
\hline Errors sources & \multicolumn{1}{c}{ Symbols } & Number \\
\hline$X$-axis & $\delta_{x x}, \delta_{y x}, \delta_{z x}, \varepsilon_{x x}, \varepsilon_{y x}, \varepsilon_{z x}$ & $1,2,3,4,5,6$ \\
$Y$-axis & $\delta_{x y}, \delta_{y y}, \delta_{z y}, \varepsilon_{x y}, \varepsilon_{y y}, \varepsilon_{z y}$ & $7,8,9,10,11,12$ \\
$Y$-axis & $\delta_{x z}, \delta_{y z}, \delta_{z z}, \varepsilon_{x z}, \varepsilon_{y z}, \varepsilon_{z z}$ & $13,14,15,16,17,18$ \\
$B$-axis & $\delta_{x b}, \delta_{y b}, \delta_{z b}, \varepsilon_{x b}, \varepsilon_{y b}, \varepsilon_{z b}$ & $19,20,21,22,23,24$ \\
Interaxis & $\gamma_{x y}, \beta_{x z}, \alpha_{y z}, \alpha_{y b}, \gamma_{y b}, \delta_{z b y}$ & $25,26,27,28,29,30$ \\
\hline
\end{tabular}

$$
\begin{aligned}
& \xi_{x}=\left[\begin{array}{c}
v_{x} \\
0_{3 \times 1}
\end{array}\right], \\
& v_{x}=\left[\begin{array}{lll}
1 & 0 & 0
\end{array}\right] \text {, } \\
& \xi_{y}=\left[\begin{array}{c}
v_{y} \\
0_{3 \times 1}
\end{array}\right] \text {, } \\
& v_{y}=\left[\begin{array}{lll}
0 & 1 & 0
\end{array}\right] \text {, } \\
& \xi_{z}=\left[\begin{array}{c}
v_{z} \\
0_{3 \times 1}
\end{array}\right] \text {, } \\
& v_{z}=\left[\begin{array}{lll}
0 & 0 & 1
\end{array}\right] \text {, } \\
& \xi_{b}=\left[\begin{array}{c}
-\omega_{b} \times q_{b} \\
\omega_{b}
\end{array}\right], \\
& \omega_{b}=\left[\begin{array}{lll}
0 & 1 & 0
\end{array}\right]^{\mathrm{T}} \text {, } \\
& q_{b}=\left[\begin{array}{lll}
0 & 0 & 0
\end{array}\right] \text {, } \\
& {\left[q_{b w}(0)\right]=\left[\begin{array}{cccc}
1 & 0 & 0 & 0 \\
0 & 1 & 0 & 0 \\
0 & 0 & 1 & D_{w r} \\
0 & 0 & 0 & 1
\end{array}\right],} \\
& {\left[g_{b t}(0)\right]=\left[\begin{array}{cccc}
1 & 0 & 0 & 0 \\
0 & 1 & 0 & 0 \\
0 & 0 & 1 & D_{t r} \\
0 & 0 & 0 & 1
\end{array}\right] \text {, }}
\end{aligned}
$$

where $D_{w r}=90 \mathrm{~mm}$ and $D_{t r}=1090 \mathrm{~mm}$ are the respective distance between the workpiece and cutting tool to the reference coordinate system in the $Z$ direction.
The general kinematics model of a multiaxis CNC machine tool based on a global coordinate system is established. The above modelling process does not need to establish a local coordinate system on each motion axis or analyse the transformation relationship between adjacent coordinate systems. It needs only the topological information of each motion axis in the global coordinate system, and then the forward kinematics model according to the kinematics chain order of the machine tool can be established efficiently.

\section{Geometric Error Analysis Modelling}

The geometric errors vary due to the wearing of machine components as the machine tool is running. In addition, complicated coupling exists among the geometric error parameters, resulting in a nonlinear distribution of geometric error terms $[6,17]$. Within those factors, the volumetric position and orientation accuracy show nonstationary time varying characteristics, and the accuracy retentivity of geometric accuracy inevitably varies with fluctuating effects at different moments. The measuring geometric error directly reflects the geometric accuracy: the higher the measurement error is, the lower the precision of the machine tool will be, and the geometric accuracy is one of the most important accuracy indexes [6, 23]. Geometric errors can affect the shape, size tolerance, and surface roughness of the machined parts $[8,42]$; for instance, a parallelism error between the spindle and guide rail will lead to tapering and poor surface roughness of the workpiece.

3.1. Accuracy Retentivity Model. Machine tool accuracy retentivity is defined as the ability of each accuracy index of the machine tool to remain within the required range for a long time under normal operation [29, 37]. The accuracy indexes of machine tools are comparatively steady over short times and show a nonlinearly decreasing tendency over a long period of time. The geometric accuracy is one of the most important accuracy indexes $[41,42]$ and is also the foundation of the working accuracy and motion accuracy of machine tools. Therefore, the dynamic evolution over time should be considered when conducting maintenance and geometric error compensation. The geometric accuracy also acts as a quasistatic accuracy that gradually declines over time and thus shows strong time-correlation behaviour [33]. In this paper, the geometric error of dynamic change is mapped to the time dimension and transformed into a timeindependent index. Then, the static index evaluation method is used to evaluate the mapped accuracy index.

The precision index of error varies nonuniformly during the adjacent two detection periods. To obtain discrete algebraic values for different times, geometric error identification should be performed after periodic measurement with a special measuring instrument. The geometric errors are mapped to the time domain, and a discrete model is established. The functional relationship between the geometric error and time $t$ is 


$$
\begin{aligned}
e_{u}= & e_{u i}+f\left[e_{u i}, e_{u i+1}\right]\left(t_{i+1}-t_{i}\right) \\
& +f\left[e_{u i}, e_{u i+1}, e_{u i+2}\right]\left(t-t_{i}\right)\left(t-t_{i+1}\right) \\
& +f\left[e_{u i}, e_{u i+1}, e_{u i+2}, e_{u i+3}\right]\left(t-t_{i}\right)\left(t-t_{i+1}\right)\left(t-t_{i+2}\right),
\end{aligned}
$$

where $f$ is the Newton interpolation function, $e_{u}$ is the function expression for geometric error terms, $u$ is the sequence number of the geometric error terms, which is listed in Table 1, and $i$ is the $i$-th time node of the geometric error measurement.

The mean of the geometric error terms in two adjacent detection periods can be expressed as

$$
\overline{e_{u}}=\frac{1}{t_{n}} \sum_{i=1}^{n} \int_{t_{i}}^{t_{i+2}} e_{u} \mathrm{~d} t .
$$

The signal-to-noise ratio can describe the influence of the geometric error terms on the accuracy of the machine tool based on the robust design method [43]. To express the ability to maintain the initial state of geometric accuracy, the fluctuation in the geometric error is also taken into account, and the accuracy retentivity of the machine tool accuracy index at time $t$ is as follows:

$$
r_{a u}=10 \lg \left(\frac{1}{{\overline{e_{u}}}^{2}+e_{v}^{2}}\right),
$$

where ${\overline{e_{u}}}^{2}$ and $e_{v}^{2}$ are the square of the mean value and the variance in the geometric error, respectively, and can be determined with equations (18) and (20):

$$
e_{v}^{2}=\frac{1}{n}\left[\left(e_{x 1}-\overline{e_{u}}\right)^{2}+\left(e_{x 2}-\overline{e_{u}}\right)^{2}+\cdots+\left(e_{x n}-\overline{e_{u}}\right)^{2}\right] .
$$

Experimental results show that the geometric error is Gaussian distributed [37, 44]. The larger the values of accuracy retention are, the smaller the fluctuations in the geometric error are, and when the geometric accuracy is lower than the failure accuracy, replacement of parts or implementation of compensation must be conducted. The position and orientation of the machine tool change with the position space of the cutting tip. The geometric error vector $\mathbf{P}_{k}, \mathbf{O}_{k}$ $(k=x, y, z)$ can be determined according to equation (12), and the mean values of the geometric error are a function of time $t$ :

$$
\left\{\begin{array}{l}
\overline{\mathbf{P}}_{k}=\frac{\iiint \int \mathbf{P}_{k} \mathrm{~d} x \mathrm{~d} y \mathrm{~d} z \mathrm{~d} \theta_{b}}{I}, \\
\overline{\mathbf{O}}_{k}=\frac{\iiint \int \mathbf{O}_{k} \mathrm{~d} x \mathrm{~d} y \mathrm{~d} z \mathrm{~d} \theta_{b}}{I},
\end{array}\right.
$$

where $I$ is space constructed by travel of tool tip.

After adding all the mean values of the geometric error vector into equation (21), the accuracy retentivity of the machine tool in a spatial position is as follows:

$$
r_{a i}=10 \lg \left(\frac{1}{\overline{\mathbf{E}}^{2}+\mathbf{E}^{2}}\right), \quad\left(\mathbf{E}=\mathbf{P}_{k}, \mathbf{O}_{k}\right) .
$$

Based on the abovementioned analysis method, the accuracy retentivity of six geometric error vectors in the workspace of the machine tool can be quantifiably expressed.

\subsection{Key Parameter Identification Modelling Based on Feature} Extraction. The parametric correlation and coupling of the geometric error terms have a great influence on the fluctuations in the position and orientation vectors, and the strength of the influence is determined by the magnitude of relevance of the geometric error terms. In addition, in the geometric error identification model, all geometric errors are usually assumed to be independent and have a consistent effect on the error vector, but the truth is quite the opposite. Error vectors have different sensitivities to geometric errors, although the relevancy coefficient of the geometric error with respect to the error vector is the same. Therefore, sensitivity evaluation should be conducted through a supplementary analysis on the basis of evaluating the relevancy and similarity between the retentivity of the geometric error terms and error vectors [39], and the relevancy, similarity of the geometric errors, and sensitivity of the error vectors corresponding to the geometric error terms should be synthetically considered. Then, key geometric error terms can be identified, and a sensitivity analysis can be included within the model training process of the embedded method of feature extraction $[26,45]$.

The correlation can be quantified by the uncertainty of the effect caused by the geometric error terms. The method of feature selection suitable for the analysis of a complex correlation relationship $[46,47]$ can effectively identify the key parameters affecting the fluctuations in geometric accuracy. The steps for identifying key geometric error terms based on feature selection are as follows:

(1) First, an evaluation criterion Eva $\left(e_{u}\right)$ is defined to evaluate the relevance between the key subset $S_{i}$ of the geometric error and the error vector. The parameter subset $S_{j}$ is an empty set, and the candidate parameters $e_{u}$ are the initial values of the identified parameters. The candidate parameters are filtered by the relevancy between the candidate parameters $e_{u}$ and error vector $\mathbf{E}$, which consists of $\mathbf{P}_{k}$ and $\mathbf{O}_{k}$. The parameters with maximum correlation, maximum sensitivity, and minimum similarity are selected as key parameters by evaluating three kinds of correlation relations when the nonempty subsets of the key parameters $k_{j}$ are nonnull. The relevant relation involved in the above problems is as follows:

$$
\operatorname{Eva}\left(e_{u}\right)=\psi_{e_{u}}^{E} R e-\vartheta_{e_{u}}^{s} S i+(1-\psi-\vartheta)\left(\operatorname{Se}\left(e_{u}, \mathbf{E}, S_{i}\right)\right) \text {, }
$$

where ${ }_{e_{u}}^{E v} R e$ is the correlation between the geometric error terms and accuracy retentivity, ${ }_{e}^{s} S i$ is the similarity between the geometric error terms and accuracy retentivity, and $\operatorname{Se}\left(e_{u}, \mathbf{E}, S_{j}\right)$ is the sensitivity of the error vector with respect to the geometric error terms. 
$\psi$ and $\vartheta$ are the weights of the characteristics, and $\psi+\vartheta \in[0,1]$.

The effect on the fluctuation in accuracy retentivity induced by the geometric error terms can be represented in relevancy, which can be expressed with the mutual information of the above parameters, as follows:

$$
{ }_{e_{u}}^{E} R e=I\left(e_{u} ; \mathbf{E}\right),
$$

where the relevancy between two random variables $\mathbf{E}\left(\mathbf{P}_{k}, \mathbf{O}_{k}\right)$ (equation (12)) and $e_{u}$ (equation (17)) is described with mutual information. This parameter represents the amount of information about one random variable contained in another random variable. According to mutual information theory, the mutual information of two random variables $x$ and $y$ is

$$
I(x ; y)=-\sum_{i=1}^{n} \sum_{j=1}^{n} p\left(x_{i}, y_{i}\right) \log 2^{p\left(x_{i}, y_{i}\right) / p\left(x_{i}\right) p\left(y_{i}\right)},
$$

where $x=\left\{x_{1}, x_{2}, x_{3}, \ldots, x_{n}\right\}, y=\left\{y_{1}, y_{2}, y_{3}, \ldots, y_{n}\right\}, p$ $\left(x_{i}, y_{i}\right)$ is the probability when $x$ equals $x_{i}$ and $y$ equals $y_{i}$ simultaneously, $p\left(x_{i}\right)$ is the probability when $x$ equals $x_{i}, p\left(y_{i}\right)$ is the probability when $y$ equals $y_{i} \cdot p\left(x_{i}, y_{i}\right)=p\left(x_{i}\right) \cdot p\left(y_{i}\right)$ when the variables $x$ and $y$ are independent, and the general relationship of $I(x, y)$ equalling 0 is established. This means that the same information does not exist in the variables $x$ and $y$.

(2) Second, the information similarity within the key parameter subset increases when there is similarity between the current parameter $e_{u}$ and the key parameter subset; therefore, the parameter similarity should be quantitatively evaluated based on correlation analysis. The similarity of parameters can be determined by evaluating information between current parameters and the subset of selected key parameters. Let $r_{u}$ be the retentivity index of the geometric error term that is contained within the corresponding vectors $\mathbf{P}_{k}$ and $\mathbf{O}_{k}$; then, the average value avg $\left(r_{u}\right)$ of the error terms that contain $N$ samples can be expressed as

$$
\operatorname{avg}\left(r_{u}\right)=\frac{1}{N} \sum_{i=1}^{N} r_{u}(t)
$$

where $r_{u}(t)$ is the $j$-dimensional feature of the $t$-th sample.

The number of error terms $e_{u}$ is $N$; then, the characteristic mean of $e_{u}$ is avg $\left(e_{u}\right)$, which can be expressed as follows:

$$
\operatorname{avg}\left(e_{u}\right)=\frac{1}{N} \sum_{j=1}^{N} e_{u}(t)
$$

where $e_{u}(t)$ is the characteristic value of the $t$-th sample. The inner-class distance of the retentivity index of the geometric error terms is

$$
\sigma_{\text {in }}^{2}(u)=\sum_{t=1}^{M}\left|r_{u}(t)-\operatorname{avg}\left(r_{u}\right)\right|^{2} .
$$

The interclass distance of the $u$-dimensional feature of the error terms is

$$
\sigma_{\text {it }}^{2}(u)=\sum_{u=1}^{m}\left|\operatorname{avg}\left(r_{u}\right)-\operatorname{avg}\left(e_{u}\right)\right|^{2} .
$$

The similarity with respect to parameter $e_{u}$ and subset $S_{j}$ of the key parameters that contain $J$ parameters is defined as follows:

$$
e_{u}^{s} S\left(e_{u}\right)=\frac{\sigma_{\mathrm{it}}^{2}(u)}{\sum_{u=1,2, \ldots, n} \sigma_{\mathrm{in}}^{2}(u)} .
$$

The above formula can be represented by the similarity of the error terms; the larger $e_{u}^{s} S\left(e_{u}\right)$ is, the lower the similarity of the features is.

(3) Third, the decline in varying accuracy retentivity due to the combined effects of the exterior environment and internal factors, which includes the interaction between the geometric error terms and error vector, and this relationship also has a great effect on the precision of machine tools. The fluctuation in the geometric error terms leads to changes in the error vectors, and the total output variance can be decoupled into the sum of variances of input parameters [48]. The variance decomposition of the error vector is as shown:

$$
V\left(e_{u}\right)=\sum_{i} V_{i}+\sum_{i} \sum_{i<j} V_{i j}+\cdots+V_{12, \ldots, n},
$$

where $V\left(e_{u}\right)$ is the total variance, which is caused by the variances of all the error parameters. $V_{i}$ is the sum of the variances that influence the various geometric errors. The interactions of the geometric errors are characterized as second order and higher order, and terms of higher order can be ignored in the case of lower orders of magnitude.

The sensitivity coefficient of each geometric error parameter is determined by

$$
S_{u}=\frac{V_{i}}{V\left(e_{u}\right)} .
$$

The effect of the geometric error on the geometric error vector and spatial accuracy of the machine tool can be accurately reflected by the sensitivity coefficients.

(4) Fourth, 30 geometric error terms and 6 vectors of position and orientation are considered in the process of key parameter identification, so the 
timeliness of key parameter identification becomes one of the key properties of the algorithm; therefore, a filter-based key parameter identification method is designed to improve the efficiency of parameter identification. The assessment method is proposed to verify whether the current subset of key parameters meets the filtering requirements. The mean weight of each feature is obtained by calculating the correlation between each geometric error and coefficient of accuracy retentivity in the original feature set based on relevancy and similarity, and irrelevant parameters with respect to accuracy retentivity (i.e., those for which the mutual information is equal to zero) are eliminated from the set of candidate parameters, letting an initial empty set be $Q$ for the set of candidate parameters $U$, and one feature is chosen from $U$ and inserted into $Q$. The approximation is achieved by maximizing the similarity characteristics of the parameters in the subset during the calculation of the similarity information:

$$
g_{u}=\arg \max _{1 \leq u \leq n}\left\{\overline{e_{u}^{s} S\left(e_{u}\right)}\right\},
$$

where $g_{u} \in Q$. For unselected features in $U$, if the chosen $g_{u}$ satisfies the relation $I\left(e_{u}\right)=I\left(g_{u}\right)=I\left(e_{u}, g_{u}\right)$ and if $e_{u}$ and $g_{u}$ are almost entirely similar, then the $g_{u}$ value is removed from $U$. Otherwise, the similarity is expressed by the maximum value $I_{\max }\left(e_{u}, g_{u}\right)$ of the mutual information of both.

The importance of features is evaluated by the criterion of maximum correlation and minimum similarity, and the most important feature is selected and inserted into set $Q$. The evaluation formula is as follows:

$$
J\left(e_{u}\right)=\frac{\overline{e_{u}^{s} S\left(e_{u}\right)}}{I_{\max }\left(e_{u}, g_{u}\right)}
$$

where the $l$-th feature of $Q$ is the basis for selection, which is expressed as follows:

$$
g_{l}=\arg \max 1 \leq u \leq n\left\{J\left(e_{u}\right)\right\} .
$$

(5) Fifth, based on the conditional mutual information, similarity evaluation, and sensitivity analysis method, the process details of the key parameter filtering method of the geometric error are shown in Figure 3.

(1) To initialize the weight coefficient, let $\psi=0$, and let $Q$ be a subset of candidate parameters.

(2) To initialize the weight coefficient $\vartheta$, the initial value is set to 0 .

(3) Calculate the evaluation criterion Eva $\left(e_{u}\right)$ of all candidate geometric error terms; the candidate geometric error terms are sorted from high to low according to the values of Eva $\left(e_{u}\right)$. The mutual information values $I\left(e_{u} ; \mathbf{E}\right)$ of all geometric error terms and error vectors are calculated.
(4) After initializing the counter variables $i$ and emptying all the geometric error terms in the subset of the key parameters, the $i$-th geometric error term is selected as the key parameter, and the similarity information value $e_{u}^{s} S\left(e_{u}\right)$ of the parameter subset and the error vector is calculated. Moreover, the counter variables are assigned as $i=i+1$.

(5) When the parameter relations meet the evaluation criterion shown in equation (23) or the counter variables $i$ are greater than the number of parameters in the set of selected geometric error terms, then proceed directly to Step 6; otherwise, return to Step 4.

(6) All key parameters of the filtered subsets are saved, and the weight coefficients are assigned by $\vartheta=\vartheta+0.1$. When the weight coefficient is established as $\psi+\vartheta>1$, the system returns to Step 3, the weight coefficient $\psi$ is assigned by $\psi=\psi+0.1$, and the calculation process returns to Step 2. Otherwise, in the process of parameter filtering, all weight coefficients of $\psi$ and $\vartheta$ are optimized by the values of the mutual information value $I\left(e_{u} ; \mathbf{E}\right)$, and the corresponding key parameters are identified, which seriously affects the accuracy of the machine tool.

\section{Case Study}

In this paper, the TGK46100 precision horizontal coordinate boring machine is used as an example for analysis and modelling. This four-axis machine tool is composed of linear axes, a headstock, a rotary axis and a worktable, which is consistent with the schematic diagram of the machine structure shown in Figure 2 in Section 2, and its technical parameters are listed in Table 2. The measurement site of the geometric errors is shown in Figure 4. To reduce the influence of the environment on the measurement results, in the experiment, the ambient temperature is controlled at $20 \pm 2^{\circ} \mathrm{C}$ with an air conditioning device, and the relative humidity is $60 \pm 10 \% \mathrm{RH}$. A Renishaw QC20-W type double ball bar (DBB) is adopted for measuring the geometric error of the rotary axis. The measurement accuracy of the DBB is $\pm(0.7+L \cdot 0.3 \%) \mu \mathrm{m}$, where $L$ stands for the length covered by the measurement and the nominal length of $L$ is $100 \mathrm{~mm}$. In addition, the feed speed of the motion is $500 \mathrm{~mm} / \mathrm{min}$ to reduce the impact of the servo error and thermal error.

4.1. Geometric Error Measurement. The geometric errors of the linear axis and rotary axis can be measured with special measuring instruments, namely, a laser interferometer measurement system (LIM), plane grating, and DBB, which are recommended by ISO 230-1, and some feasible measurement methods are presented. The 10-line method needs to measure only the positioning errors of nine motion lines and one body diagonal by the synchronous motion of three axes [49]. The straightness errors need not be measured directly, and this approach also involves fewer measuring 


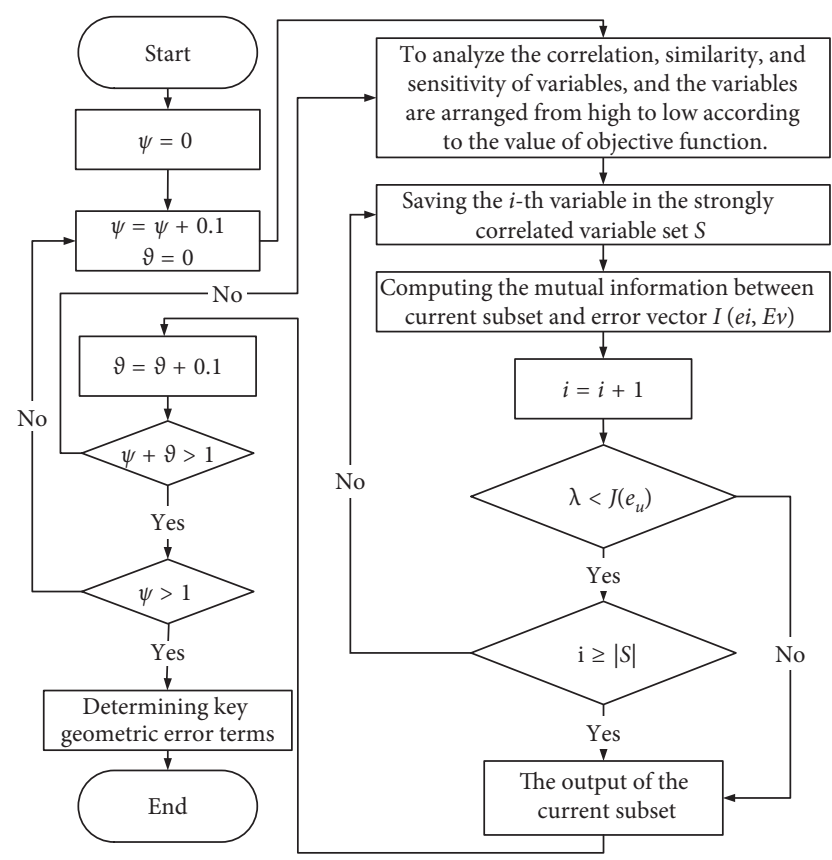

FIgURE 3: The flow chart of the key parameter recognition method.

TABLe 2: Technical parameters of the four-axis machine tool.

\begin{tabular}{|c|c|}
\hline $\begin{array}{l}\text { Technical } \\
\text { indexes }\end{array}$ & Specifications \\
\hline $\begin{array}{l}\text { Stroke of the linear } \\
\text { axes }(X-, Y-, \text { and } Z \text {-axes })(\mathrm{mm})\end{array}$ & 1000,900 , and 900 \\
\hline Stroke of the rotary axis ( $B$-axis) $\left(^{\circ}\right)$ & 360 \\
\hline $\begin{array}{l}\text { Positioning accuracy of the } \\
\text { linear axis }(\mathrm{mm})\end{array}$ & 0.004 \\
\hline $\begin{array}{l}\text { Maximum speed of the } \\
\text { linear axis }\left(\mathrm{m} \cdot \mathrm{min}^{-1}\right)\end{array}$ & 48 \\
\hline $\begin{array}{l}\text { Maximum speed of the } \\
\text { spindle }\left(r \cdot \mathrm{min}^{-1}\right)\end{array}$ & 8000 \\
\hline
\end{tabular}

lines than the 12-line method and 9-line method. In addition, the method can separate 21 geometric errors of the linear axes and is not dependent on geometric error models. Hence, the 10-line method is applied to determine the algebraic values of the linear error terms by using the Renishaw XL-80 LIM system, and error terms of the rotary axis are measured with the method described in reference [49]. Because the main region of machining movement is commonly in the central part of the guideway $[31,34]$, and based on this, the measurement range is selected as part of the stroke of linear axes.

The cubic Newton interpolation was adopted for expressing the variation in geometric accuracy according to equation (17), and the first point of the curve of geometric error can be determined after at least three measurements. This experiment of geometric error measurement was conducted every three days in the first ten days of each of 4 months for a total of 13 measurements.

Partial results of PDGEs identification are shown in Figure 5.

The identification values of PIGEs are shown in Table 3.
Figure 5(a) shows that the positioning error $\delta_{x x}$ of the PDGEs along the $X$-axis increases with an increasing $X$-axis coordinate; the deviation value of $\delta_{x x}$ is the largest among the three linear geometric errors, and the error trend is essentially linear with the position of the coordinate axis. There is a linear relationship between the error direction and the position of the motion axes. The maximum deviation values of the straightness $\delta_{x z}$ and $\delta_{x y}$ are $15.1 \mu \mathrm{m}$ and $23.4 \mu \mathrm{m}$, respectively, and the error has a nonlinear relationship with the position of the linear axis. The distribution of the three angular errors of the PDGEs along the $X$-axis is nonlinear, as shown in Figure 5(b). Figure 5(c) shows that the linear errors of the $Y$-axis increase linearly, and the maximum deviation appears at the end of the travel of the $Y$-axis. The maximum deviation of the linear displacement error is from the origin of the measurement coordinate system to $+23.5 \mu \mathrm{m}$. The average deviation of the three linear errors of the $Y$-axis is $5.3 \mu \mathrm{m}$. The three angular errors of the PDGEs of the $Y$-axis increase nearly linearly with increasing $\mathrm{NC}$ command of the $Y$-axis, as shown in Figure 5(d), and the angular error curve is concave in the range of $350-450 \mathrm{~mm}$, which indicates that the parallelism error of the guideway of the $Y$-axis may have arisen in assembly. Similarly, the distribution of the straightness error of the $Z$-axis in the vertical plane and horizontal plane is nonlinear, which indicates that the manufacturing and installation of the guideway incurred defects in the corresponding direction. The geometric errors of the $B$-axis are nonlinear along the direction of motion, the larger of which is the linear error $\delta_{z b}$ that oscillates over a wide range from $-5.5 \mathrm{~mm}$ to $+20.7 \mathrm{~mm}$, which depends on the radial runout of the worm gear ring.

The distribution of the error vector of the machine tool with position and time can be determined according to the geometric error measurements and the kinematic model in equation (12), as shown in Figure 6.

The error vectors of the four-axis horizontal boring machine are relative to the position of the motion axes. The error vector increases with increasing command position of the motion axis, and the error curve is essentially a linear function of the position. The error vector first increases with increasing operation time of the machine tool and then stabilizes, and the error curve shows a nonlinear dependency on time. Even along a specific direction of the machine tool, the error vector varies due to the movement of the other axis. Therefore, it is critical to consider the variation in accuracy retentivity of the machine tools and recognize the key geometric errors, which is very useful to improve the compensation precision essential for precision machining.

4.2. Accuracy Retentivity Evaluation. With the accuracy retentivity of the geometric error terms at time $t_{3}$ taken as an example, first, the mean value is calculated by equation (17), and the accuracy retentivity coefficient of the error term can be determined with equation (18), as shown in Figures 7 and 8 . Then, the changes of the mean values of the error vector and accuracy retentivity are calculated with equations (21) and (22). 


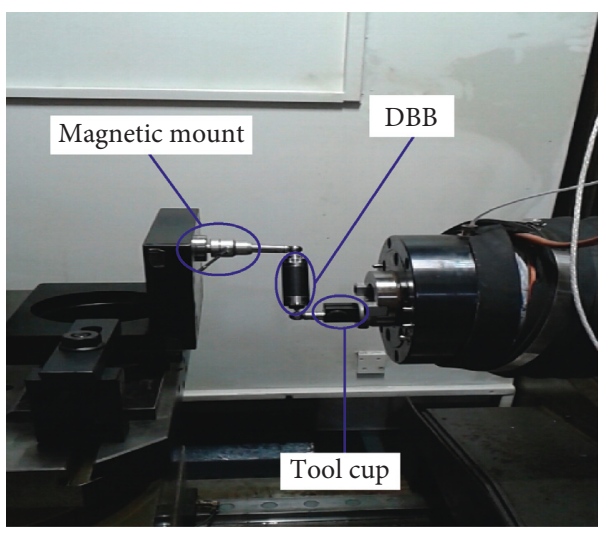

(a)

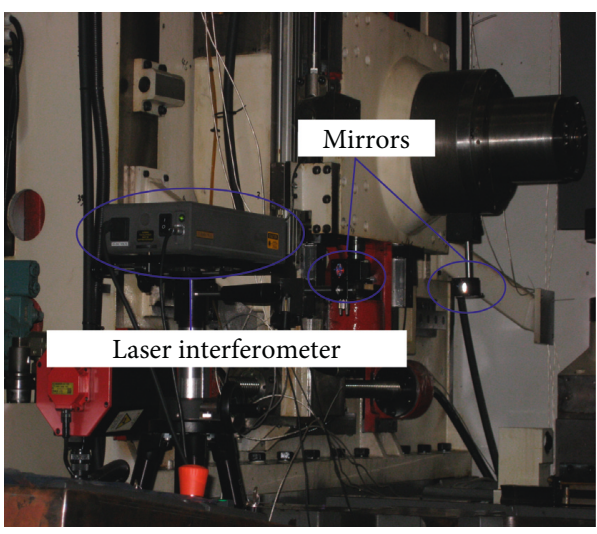

(b)

Figure 4: Measurement of 30 geometric errors for the four-axis machine tool. (a) Rotary axis measurement with a DBB. (b) Linear axis measurement with an LIM.

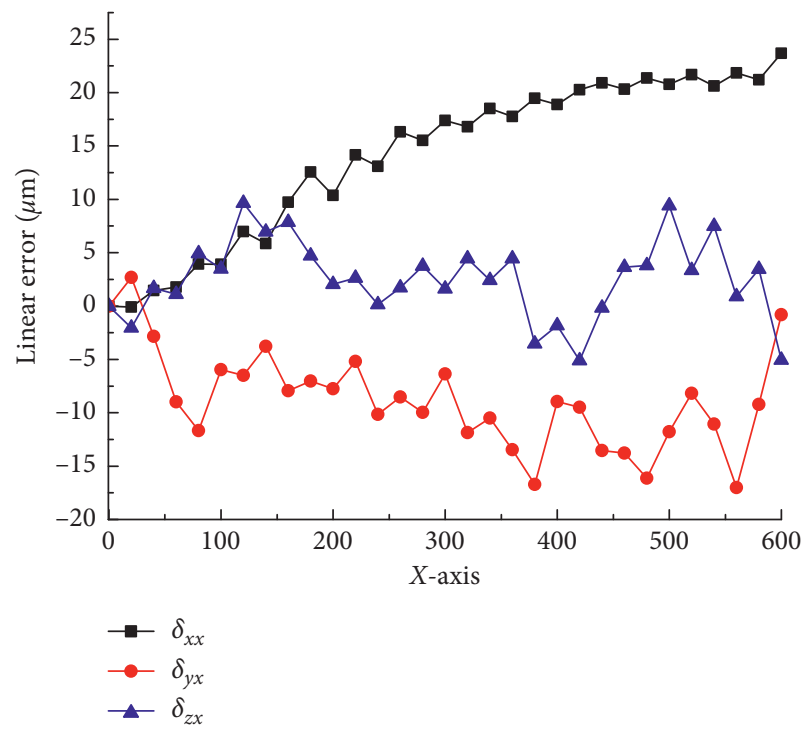

(a)

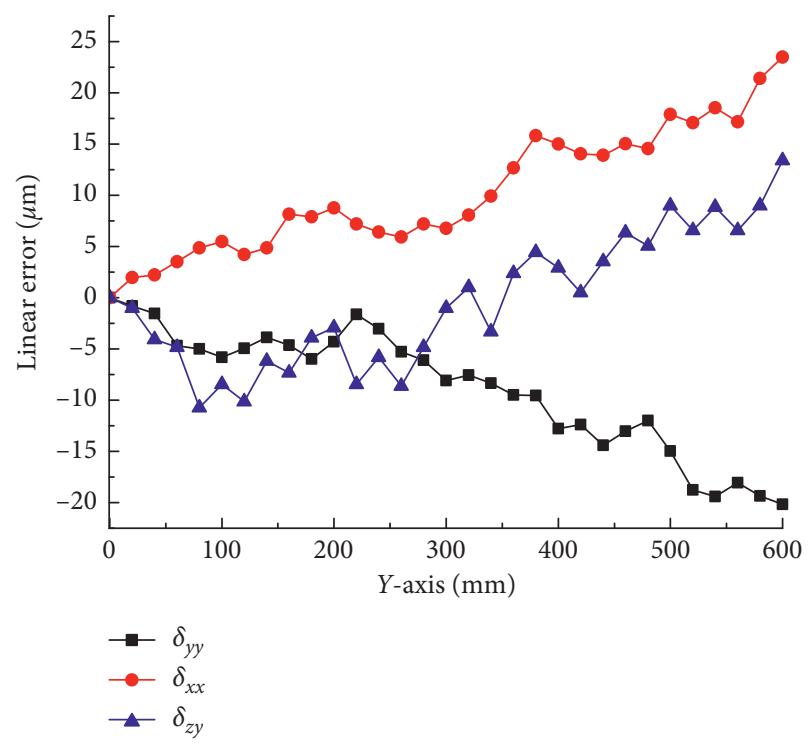

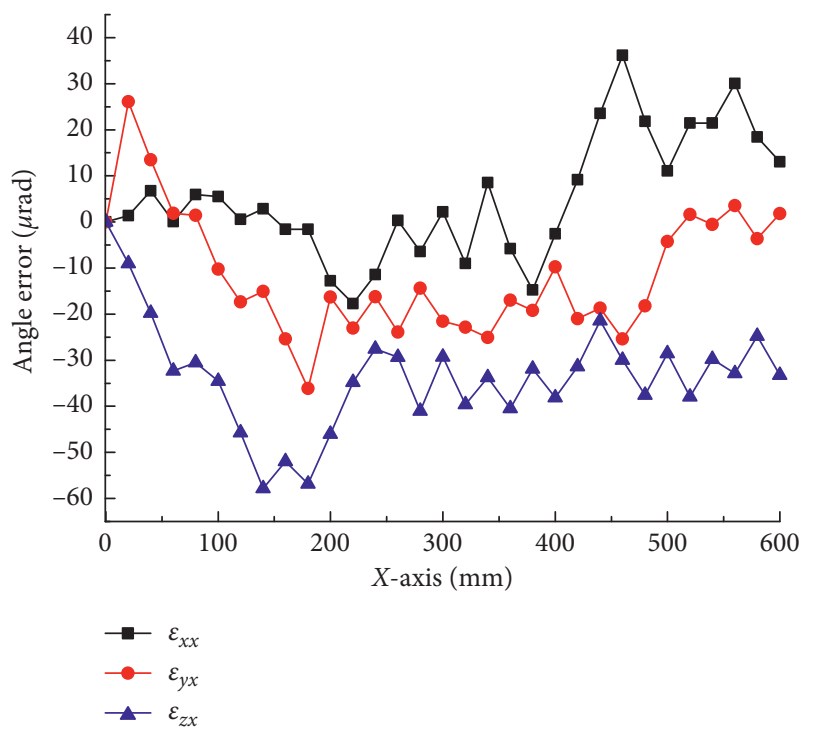

(b)

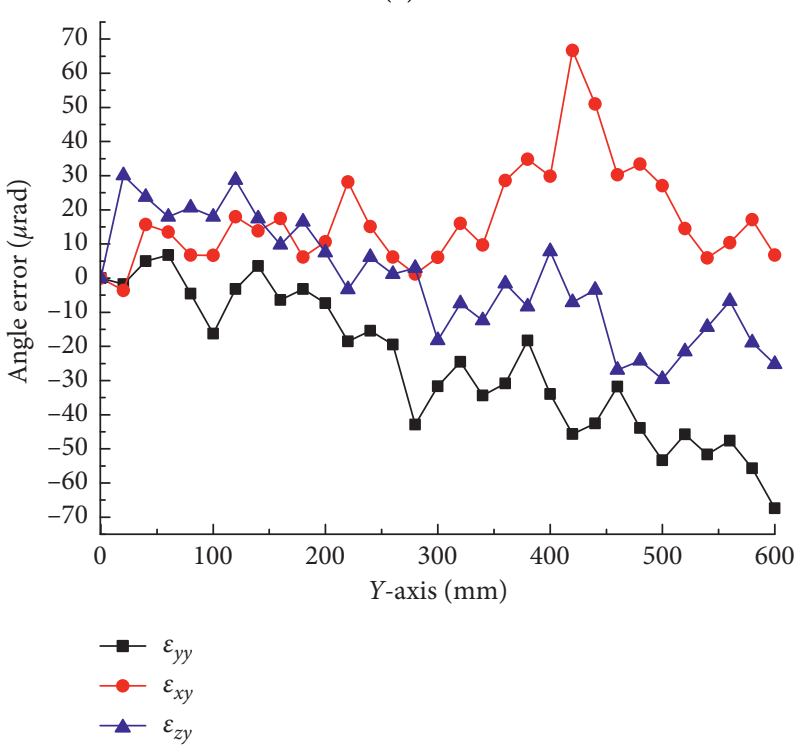

(d)

Figure 5: Continued. 


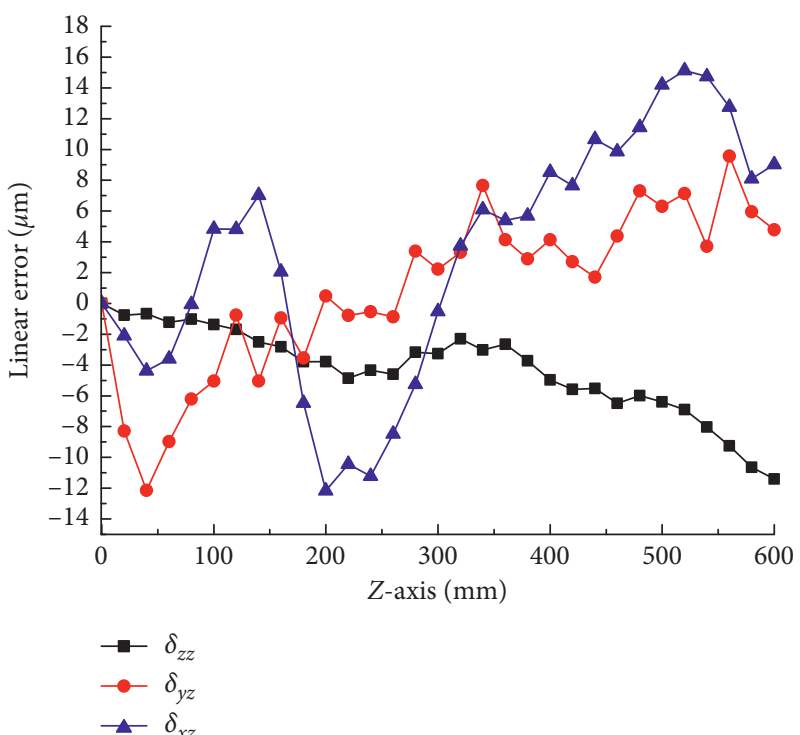

(e)

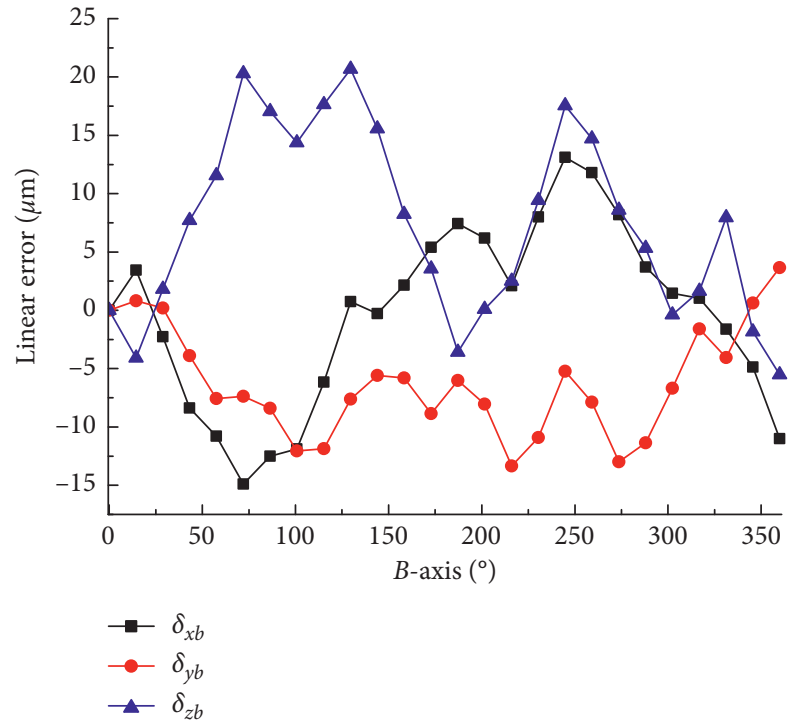

(g)

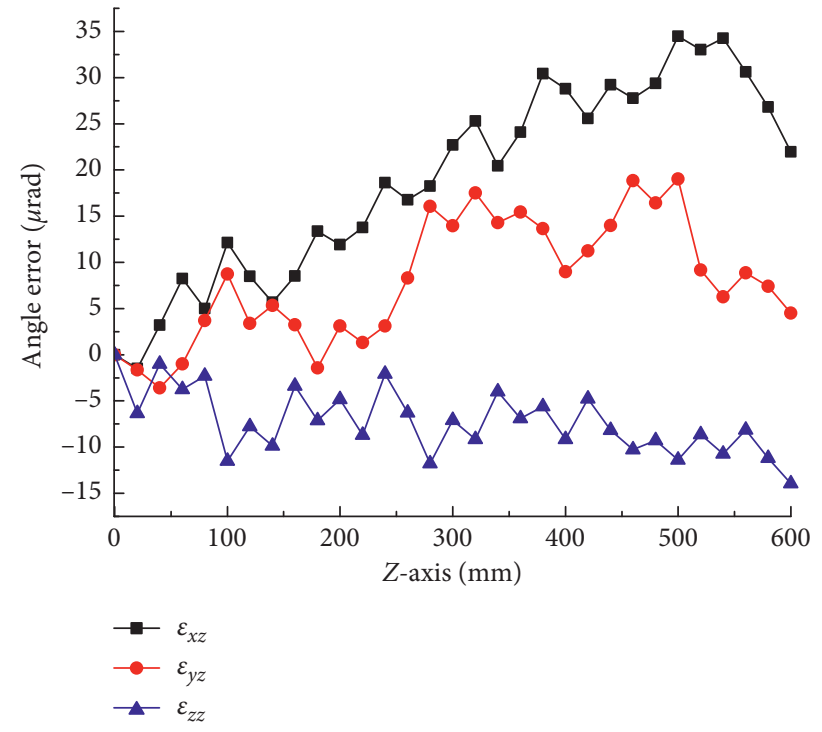

(f)

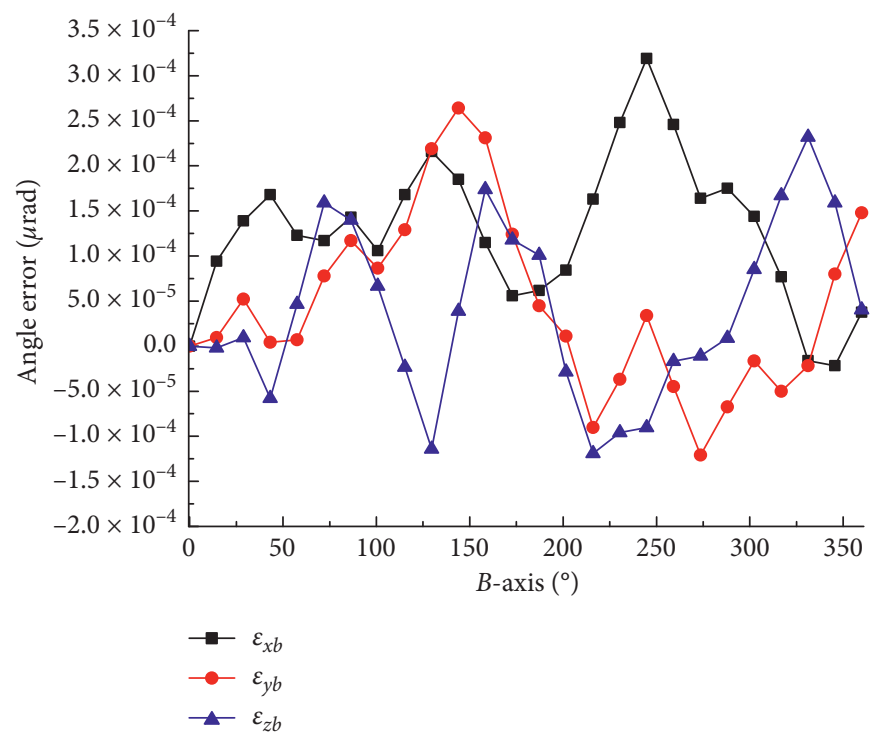

(h)

Figure 5: Identified values of geometric error. (a) Linear errors of the $X$-axis. (b) Angle errors of the $X$-axis. (c) Linear errors of the $Y$-axis. (d) Angle errors of the $Y$-axis. (e) Linear errors of the $Z$-axis. (f) Angle errors of the $Z$-axis. (g) Linear errors of the $B$-axis. (h) Angle errors of the $B$-axis.

TABLE 3: Identified values of PIGEs.

\begin{tabular}{lcc}
\hline Sequence number & Error terms & Identified values $(\mu \mathrm{m} / \mathrm{m})$ \\
\hline 25 & $\gamma_{x y}$ & 32.3 \\
26 & $\beta_{x z}$ & 33.6 \\
27 & $\alpha_{y z}$ & -16.3 \\
28 & $\alpha_{y b}$ & -19.1 \\
29 & $\gamma_{y b}$ & 10.2 \\
30 & $\delta_{z b y}$ & 12.6 \\
\hline
\end{tabular}

As shown in Figure 7, the accuracy retentivity order of the degree of influence for the geometric errors in $\mathbf{P}_{x}$ is $\delta_{x x}>\varepsilon_{z y}>\delta_{y b}>\delta_{x z}>\delta_{x b}>\beta_{x z}>\gamma_{x y}>\varepsilon_{y x}>\delta_{x y}>\varepsilon_{y y}>\varepsilon_{z x}$. The accuracy retentivity order of the degree of influence for geometric errors in $\mathbf{P}_{y}$ is $\delta_{y y}>\alpha_{y z}>\varepsilon_{z x}>\delta_{y z}>\varepsilon_{z y}>\varepsilon_{z b}>$ $\gamma_{x y}>\varepsilon_{x x}>\varepsilon_{x y}>\delta_{y x}$. The accuracy retentivity order of the degree of influence for the geometric errors in $\mathbf{P}_{z}$ is $\delta_{z z}>\delta_{y x}>\delta_{z b y}>\delta_{y z}>\varepsilon_{y z}>\varepsilon_{x y}>\delta_{x y}>\varepsilon_{x x}>\alpha_{y z}$. Among the factors that affect the accuracy retentivity of $\mathbf{P}_{x}$, the accuracy retentivity of the positioning error is relatively high, which is caused by small fluctuations in the positioning accuracy. The accuracy of the straightness along the $Y$ direction $\delta_{y x}$ in the error vector of $\mathbf{P}_{y}$ is the lowest, and the minimum value is 0.83 . The positioning accuracy along the $Z$ direction $\delta_{z z}$ in the error vector $\mathbf{P}_{z}$ is the highest, at 0.96 .

As shown in Figure 8, The mean value of the geometric accuracy retentivity of the geometric error of the $B$-axis along the $X$ direction is 0.90 , and the minimum value is 0.83 ; 


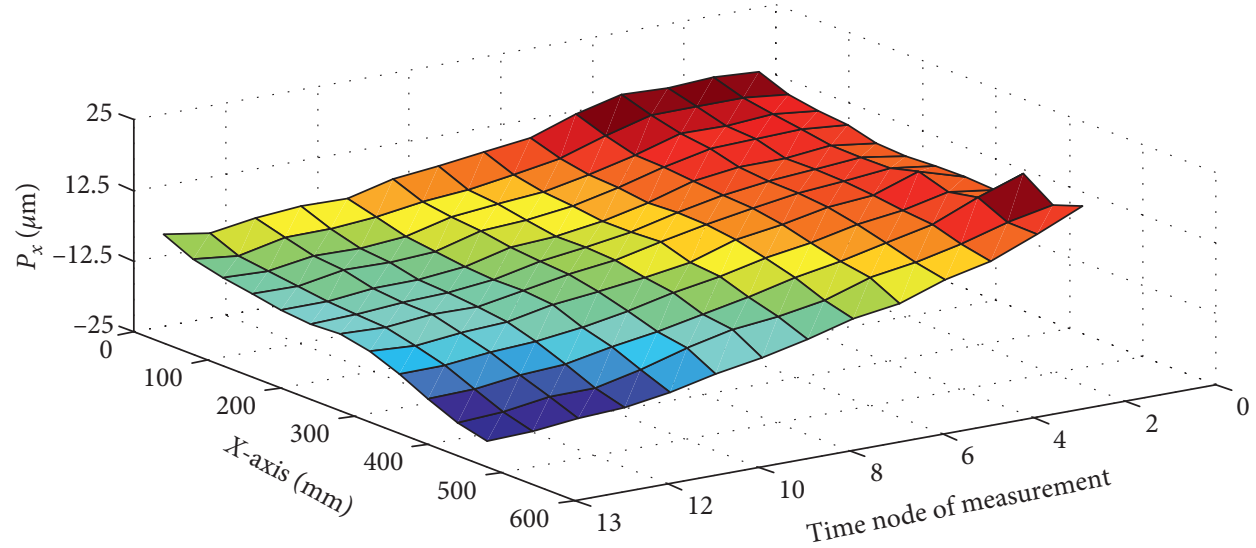

(a)

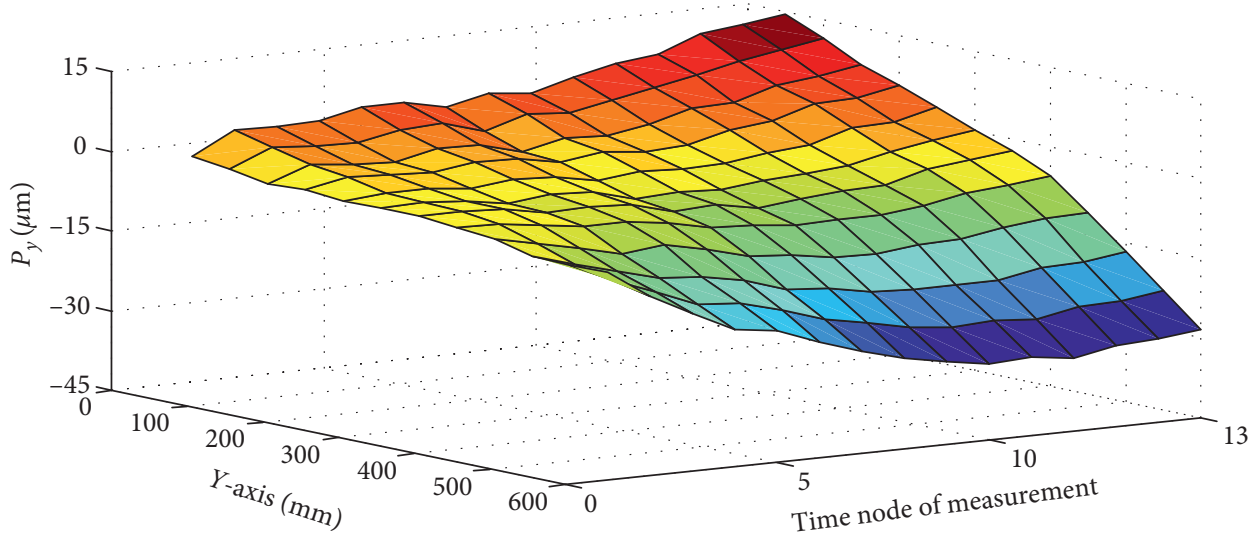

(b)

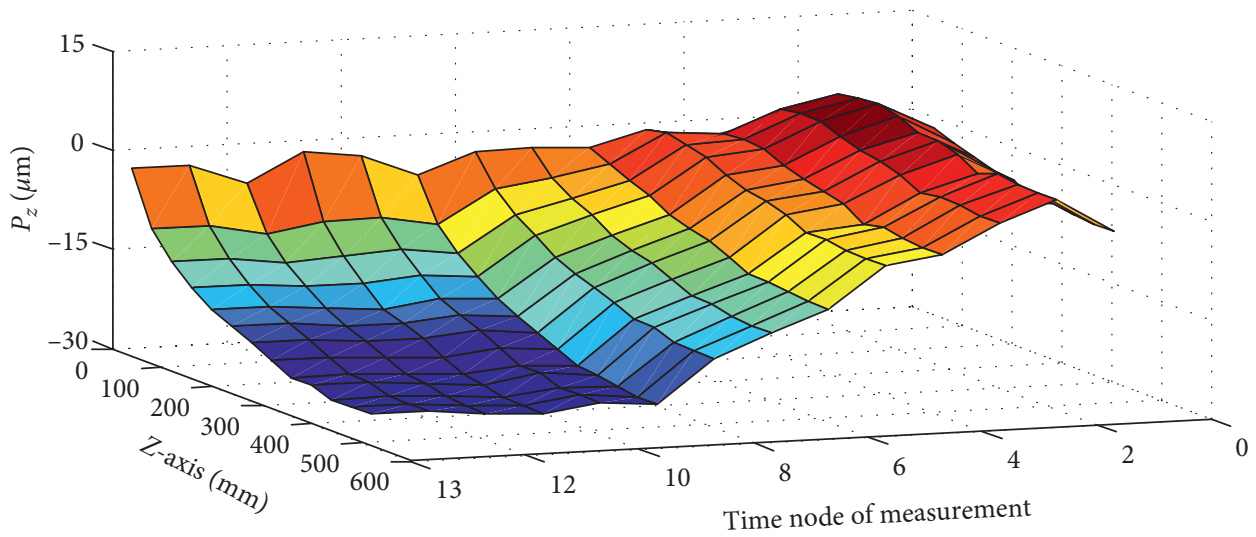

(c)

Figure 6: Continued. 


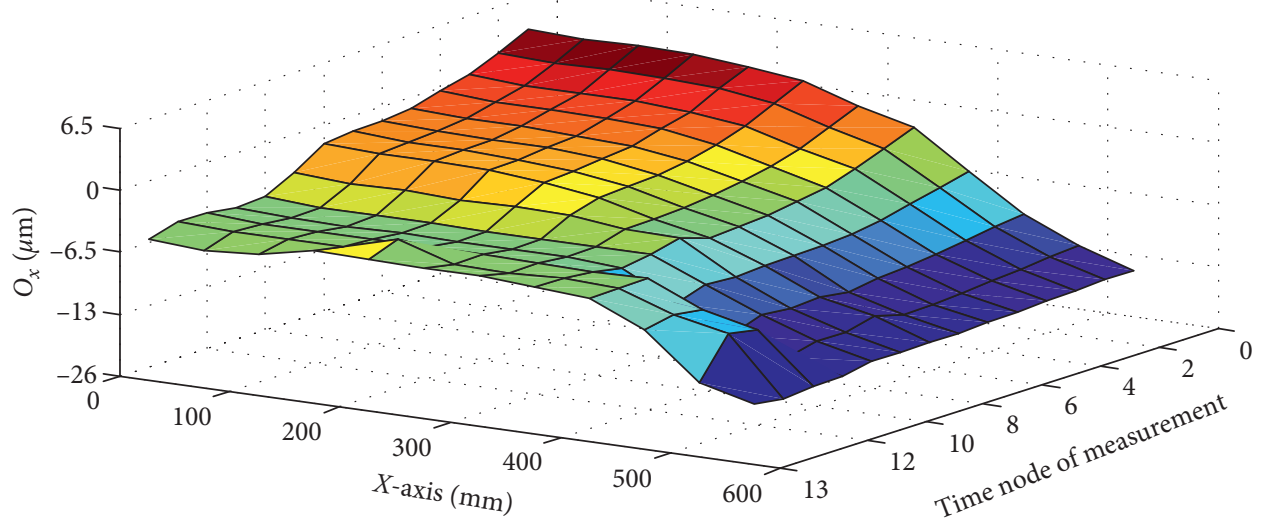

(d)

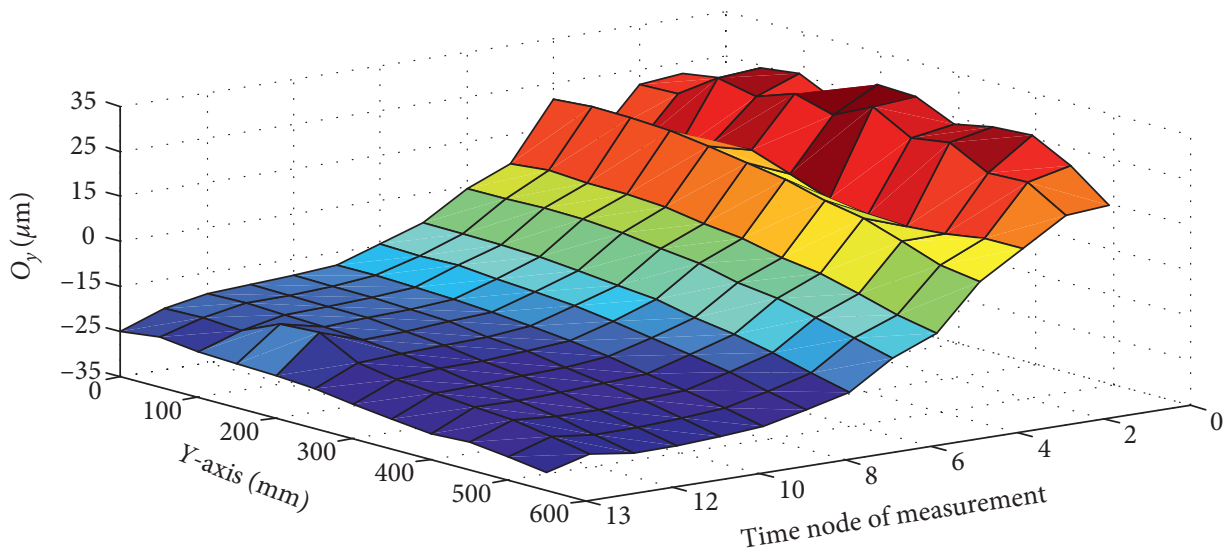

(e)

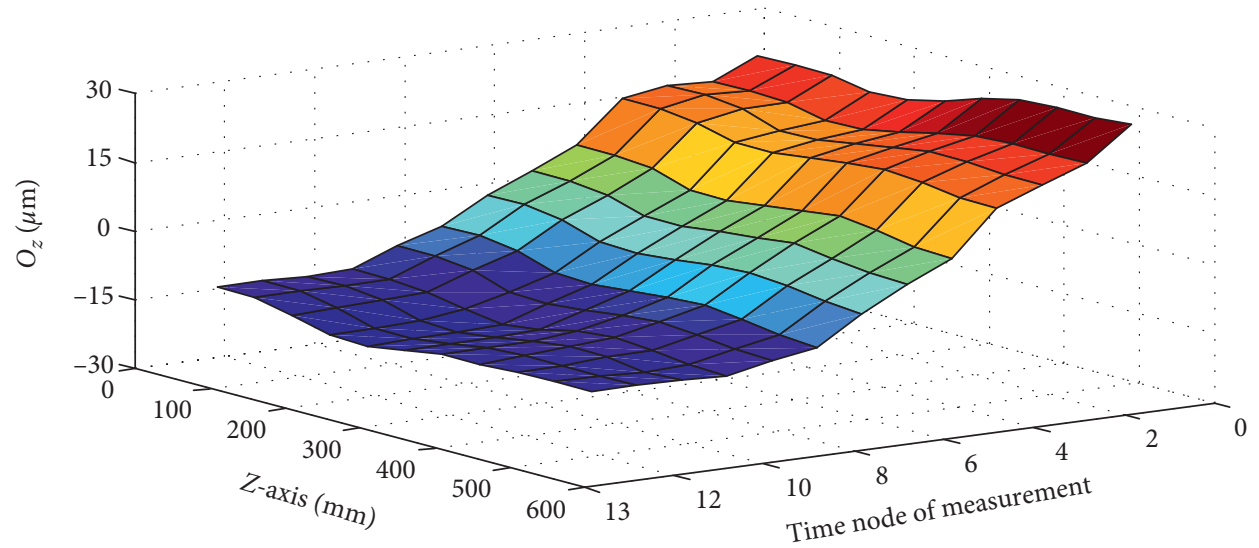

(f)

Figure 6: Distribution of the error vector with position and time. (a) Error vector $\mathbf{P}_{x}$. (b) Error vector $\mathbf{P}_{y}$. (c) Error vector $\mathbf{P}_{z}$. (d) Error vector $\mathbf{O}_{x}$. (e) Error vector $\mathbf{O}_{y}$. (f) Error vector $\mathbf{O}_{z}$.

the mean value of the geometric accuracy retentivity of the geometric error along the $Y$ direction is 0.92 , and the minimum value is 0.84 ; and the mean value of the geometric accuracy retentivity of the geometric error along the $Z$ direction is 0.90 , and the minimum value is 0.83 . The positioning error $\delta_{z y b}$, angle errors $\varepsilon_{y b}$ and $\varepsilon_{z b}$, and squareness error $\gamma_{x y}$ are the three types of angle errors with the minimum accuracy retentivity. This means that for the rotary axis, the verticality of the worm wheel and the squareness between the $X$-axis and $Y$-axis are the parameters that cause serious fluctuations in geometric accuracy retentivity.

The algebraic values of each geometric error at the measuring time $t$ are substituted into equations (12) and (22), and the accuracy retentivity of each error vector of the machine tool can be determined over the corresponding measurement time, as shown in Table 4. According to 


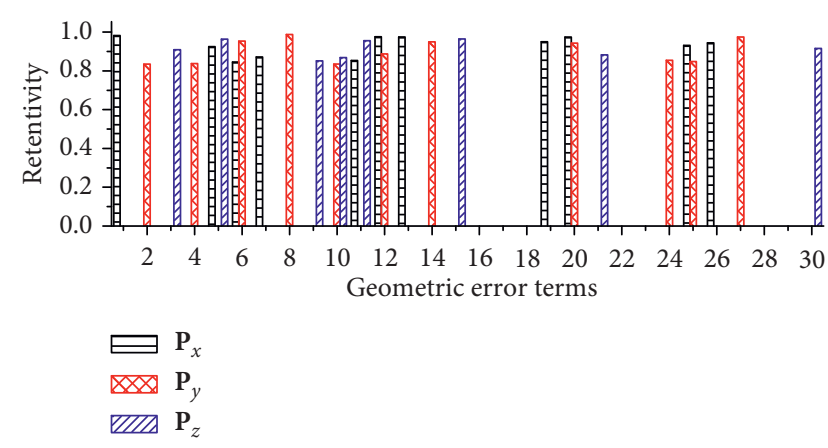

Figure 7: Accuracy retentivity analysis for the position vector at $t_{3}$.

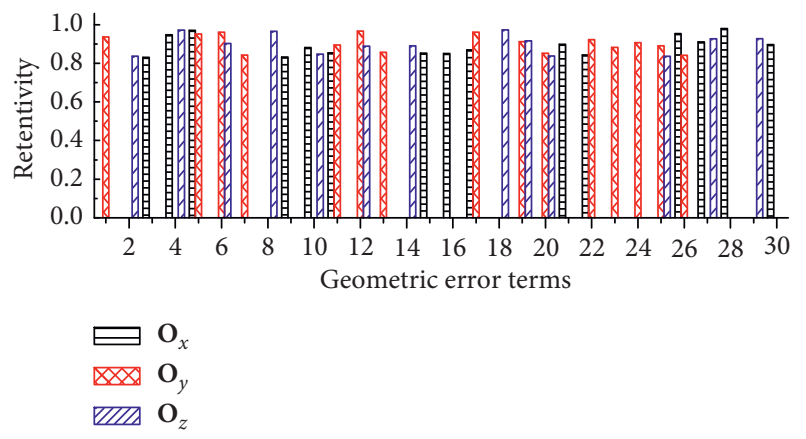

Figure 8: Accuracy retentivity analysis for the orientation vector at $t_{3}$.

TABLE 4: Analysis results of geometric accuracy retentivity.

\begin{tabular}{lcccccc}
\hline $\begin{array}{l}\text { Measurement } \\
\text { time }\end{array}$ & $r_{a p x}$ & $r_{a p y}$ & $r_{a p z}$ & $r_{a o x}$ & $r_{a o y}$ & $r_{a o z}$ \\
\hline$t_{1}$ & 0.9872 & 0.9967 & 0.9988 & 0.9803 & 0.9993 & 0.9937 \\
$t_{2}$ & 0.9774 & 0.9958 & 0.9965 & 0.9767 & 0.9719 & 0.9918 \\
$t_{3}$ & 0.9732 & 0.9819 & 0.9953 & 0.9530 & 0.9688 & 0.9808 \\
$t_{4}$ & 0.9439 & 0.9818 & 0.9914 & 0.9489 & 0.9683 & 0.9782 \\
$t_{5}$ & 0.9402 & 0.9697 & 0.9837 & 0.9349 & 0.9435 & 0.9752 \\
$t_{6}$ & 0.9238 & 0.9656 & 0.9660 & 0.9313 & 0.9317 & 0.9506 \\
$t_{7}$ & 0.9170 & 0.9413 & 0.9300 & 0.9301 & 0.8887 & 0.9499 \\
$t_{8}$ & 0.8982 & 0.9406 & 0.9252 & 0.9189 & 0.8672 & 0.9270 \\
$t_{9}$ & 0.8920 & 0.9351 & 0.9159 & 0.9178 & 0.8530 & 0.9249 \\
$t_{10}$ & 0.8882 & 0.9052 & 0.9041 & 0.9113 & 0.8504 & 0.9192 \\
$t_{11}$ & 0.8752 & 0.8878 & 0.8930 & 0.9061 & 0.8463 & 0.9101 \\
$t_{12}$ & 0.8726 & 0.8849 & 0.8987 & 0.9019 & 0.8457 & 0.9084 \\
$t_{13}$ & 0.8690 & 0.8774 & 0.8808 & 0.8971 & 0.8395 & 0.8982 \\
\hline
\end{tabular}

Table 4, the geometric accuracy retentivity declines with the longer running time of the machine tool because the geometric accuracies are degraded by wear. The accuracy retentivity of the geometric error vectors decreases with time, which means that the geometric errors of the machine tool are increasing and decreases particularly rapidly in the initial phase. However, the geometric accuracy retentivity of the error vectors is relatively stable at later time periods. The geometric accuracy retentivity of the measured geometric errors is greater than zero, and the identified values are under the allowance range.
The geometric errors are not exactly equal to the initial accuracy because the machine tool has operated for some time; hence, the geometric accuracy retentivity of the error vectors is not equal to 1 at the times of the initial measurement or retention assessment. For the position error vectors $\mathbf{P}_{x}, \mathbf{P}_{y}$, and $\mathbf{P}_{z}$, the variation in the rate of accuracy retentivity is $11.97 \%, 11.97 \%$, and $11.81 \%$, respectively, and for the orientation error vectors $\mathbf{O}_{x}, \mathbf{O}_{y}$, and $\mathbf{O}_{z}$, the variation in the rate of accuracy retentivity is $8.49 \%, 15.99 \%$, and $9.61 \%$, respectively. The variation in geometric accuracy retentivity in the direction of the $Y$ - and $B$-axes is significantly larger than that in the other motion axes, and this finding is consistent with the results obtained from the identified values of geometric errors, in which the geometric errors in the $Y$-axis direction are slightly greater than those in the other directions. This result shows that the analysis results are in good agreement with the measurement results.

4.3. Critical Error Analysis. The geometric accuracy retentivity can effectively describe the variation in accuracy. However, some geometric errors have a larger sensitivity index and smaller correlation coefficients corresponding to error vectors. This means that a single indicator parameter cannot accurately evaluate the key error terms due to the use of the one-sided analysis results. To avoid this limitation, the critical geometric error terms are extracted by using the results of the geometric error similarity, geometric accuracy retentivity, and sensitivity. Since higher-order error terms contribute a negligibly small influence on the volumetric error vector, the total variance in the volumetric error in equation (31) is calculated and analysed by the sum of the first-order variances, and the coupling effect between the geometric errors is analysed with equation (23) based on relevancy and similarity, which can avoid the influence of the algebraic stack of nonlinear geometric errors in equations (12) and (31).

Based on the identification values of each geometric error of measurement results over 4 months, the Latin cube sampling method is used to determine 200 values in the interval of the maximum and minimum values during each measuring period. According to the key geometric error analysis method proposed in Section 3.2, the weight coefficients of the geometric error terms can be determined, as shown in Table 5.

As shown in Table 5, the weight coefficients between the error and error vectors can be determined with the gain ratio of the evaluation criteria. As shown in the analysis results of key geometric error identification, the mean value of the weight coefficients is 2.21 , and the minimum and maximum weight coefficients are 3.99 and 1.04, respectively. The key error terms for the error vectors of the position and orientation can be obtained based on the results of the above analysis, which are shown in Table 5.

The error terms whose weight coefficients are greater than the mean value 2.21 are classified as the key geometric error terms. The weight coefficients of the key geometric errors for the error vectors of the position and orientation in 
TABLE 5: Weight coefficients of the geometric error terms based on feature extraction.

\begin{tabular}{|c|c|c|}
\hline Error & Parameters & Weight index \\
\hline$\delta_{x x}$ & 1 & 1.23 \\
\hline$\delta_{y x}$ & 2 & 2.06 \\
\hline$\delta_{z x}$ & 3 & 1.59 \\
\hline$\varepsilon_{x x}$ & 4 & 3.84 \\
\hline$\varepsilon_{y x}$ & 5 & 1.81 \\
\hline$\varepsilon_{z x}$ & 6 & 3.99 \\
\hline$\delta_{x y}$ & 7 & 2.17 \\
\hline$\delta_{y y}$ & 8 & 2.01 \\
\hline$\delta_{z y}$ & 9 & 1.04 \\
\hline$\varepsilon_{x y}$ & 10 & 3.38 \\
\hline$\varepsilon_{y y}$ & 11 & 1.49 \\
\hline$\varepsilon_{z y}$ & 12 & 1.51 \\
\hline$\delta_{x z}$ & 13 & 1.93 \\
\hline$\delta_{y z}$ & 14 & 2.86 \\
\hline$\delta_{z z}$ & 15 & 1.50 \\
\hline$\varepsilon_{x z}$ & 16 & 3.07 \\
\hline$\varepsilon_{y z}$ & 17 & 1.47 \\
\hline$\varepsilon_{z z}$ & 18 & 1.75 \\
\hline$\delta_{x b}$ & 19 & 2.81 \\
\hline$\delta_{y b}$ & 20 & 1.79 \\
\hline$\delta_{z b}$ & 21 & 1.95 \\
\hline$\varepsilon_{x b}$ & 22 & 1.96 \\
\hline$\varepsilon_{y b}$ & 23 & 1.69 \\
\hline$\varepsilon_{z b}$ & 24 & 1.46 \\
\hline$\gamma_{x y}$ & 25 & 3.74 \\
\hline$\beta_{x z}$ & 26 & 2.48 \\
\hline$\alpha_{y z}$ & 27 & 3.62 \\
\hline$\alpha_{y b}$ & 28 & 2.19 \\
\hline$\gamma_{y b}$ & 29 & 1.93 \\
\hline$\delta_{z b y}$ & 30 & 2.05 \\
\hline
\end{tabular}

descending order are $\varepsilon_{z x}>\varepsilon_{x x}>\gamma_{x y}>\alpha_{y z}>\varepsilon_{x y}>\varepsilon_{x z}>\delta_{y z}>$ $\delta_{x b}>\beta_{x z}$, in which $\varepsilon_{z x}$ and $\varepsilon_{x x}$ are the angle errors of the $X$ axis, $\varepsilon_{x y}$ is the angle error of the $Y$-axis, $\alpha_{y z}$, $\varepsilon_{x z}$, and $\delta_{y z}$ are the geometric errors of the $Z$-axis, and $\gamma_{x y}$ and $\beta_{x z}$ are the PIGEs of the linear axes. In view of the effects of the geometric errors on the corresponding motion axis, the sums of the weight coefficients on the $X$-axis, $Y$-axis, $Z$-axis, and $B$ axis are $2.42,1.93,2.10$, and 1.94 , respectively.

As shown in Tables 4 and 5, the motion along the linear axis and the motion in the $Y$ direction of the rotary axis can significantly reduce the accuracy retentivity of the error vector $\mathbf{O}_{y}$. For example, the accuracy retentivity of the error vector $\mathbf{O}_{y}$ is most obviously reduced by $\varepsilon_{z x}, \beta_{x z}, \gamma_{x y}$, and $\delta_{x b}$ when the $B$-axis rotates, although there are noncritical geometric errors such as $\delta_{x x}, \varepsilon_{y x}, \delta_{x y}, \varepsilon_{y y}, \varepsilon_{z y}, \delta_{x z}, \varepsilon_{y z}, \delta_{x b}, \delta_{y b}$, $\varepsilon_{x b}$, and $\varepsilon_{y b}$ and although the latter occupies a large number of geometric error terms. However, the positioning accuracy is an important inspection index for machine tools according to ISO 230-1. The positioning error correlates highly with other geometric errors subject to the same error vector, which results in a total effect not being the simple algebraic stack of geometric errors and ultimately causes the accuracy of the error vectors to fluctuate slightly. For example, the positioning accuracy $\delta_{x y}$ of the linear axis is not the key error; hence, the accuracy retentivity of the error vector in its corresponding direction fluctuates slightly.

The sum of the evaluation coefficients is 2.67 , which is composed of 6 PIGEs. Clearly, the sum of the evaluation coefficients of the latter is far greater than that of the former, indicating that the effect of the PIGEs on the error vectors of the position and orientation are greater than those of the other motion axes. The PIGEs are mainly caused by assembly deviation. Table 5 shows that the effect of the geometric errors on the spatial position accuracy is related not only to the size of the errors but also to the structure of the machine tool. The above key geometric error terms should be controlled strictly in accuracy allocation to improve the maintenance characteristics, and the above analysis results are significant and instructive for improving the geometric accuracy retentivity of the multiaxis machine tool in an accuracy evaluation perspective.

\subsection{Error Compensation Based on an Optimization Method.} The compensation effect is reduced due to the coupling of geometric errors, and the optimization method is an effective means of identifying the best compensation value. The FOA (fly optimization algorithm) is an optimization algorithm based on the foraging behaviour of flies. As a new evolutionary algorithm, compared with other traditional optimization algorithms, such as the ant colony algorithm, genetic algorithm, and fish swarm algorithm, this method has the advantages of less parameter setting, simple program implementation, and fast operation speed and has been applied in various fields of science and engineering [50]. However, there are no studies that apply the FOA to geometric error compensation. This aspect needs to be studied further due to the geometric error of the four-axis horizontal boring machine having its own unique features.

In this paper, the forward kinematics equation of the machine tool is established based on equation (12), and the bidirectional conversion relationship between the NC code and the tool posture and attitude can be realized. To reduce the impact of the geometric errors on the position and posture of the cutting tool, the accuracy retentivity and the influence of fluctuation caused by geometric errors should be considered for determining the optimum value.

The flow chart of geometric error compensation based on the FOA [49], which considers the accuracy retentivity and the influence of fluctuation caused by geometric error, is shown in Figure 9. The basic steps of the FOA in this section are as follows [50]:

(1) The parameters are initialized; the population size is Spop, the maximum generation Mgen of foraging of the fruit flies is set, and the population position coordinates are randomly initialized as $\left(X_{0}, Y_{0}, Z_{0}\right)$.

(2) The directions and distances of the random search by using the olfactory systems of fruit fly individuals can be expressed as 


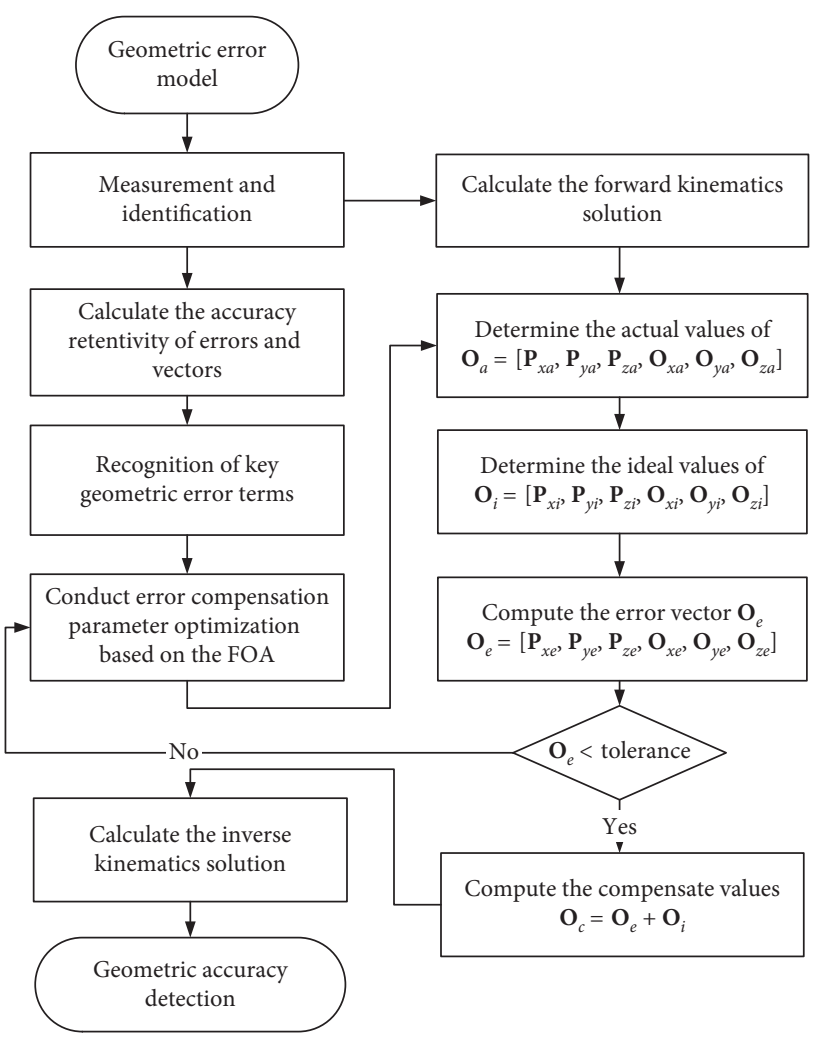

FIGURE 9: Flow chart of geometric error compensation.

$$
\left\{\begin{array}{l}
X_{i}=X_{0}+\left[L_{0}-\frac{L_{0}\left(m_{\mathrm{gen}}-1\right)}{M_{\mathrm{gen}}}\right] \times \operatorname{rand}(1,1) \\
Y_{i}=Y_{0}+\left[L_{0}-\frac{L_{0}\left(m_{\mathrm{gen}}-1\right)}{M_{\mathrm{gen}}}\right] \times \operatorname{rand}(1,1), \\
Z_{i}=Z_{0}+\left[L_{0}-\frac{L_{0}\left(m_{\mathrm{gen}}-1\right)}{M_{\mathrm{gen}}}\right] \times \operatorname{rand}(1,1),
\end{array}\right.
$$

where $i=1,2, \ldots$, Spop, $L_{0}$ is the initial step, and Mgen is the current value of foraging for the fruit flies.

(3) The distance between the current location and the coordinate origin of the first fruit fly individual needs to be estimated by equation (37), and then the evaluation value $H_{i}$ of flavour concentration is

$$
\begin{aligned}
\text { Dist }_{i} & =\sqrt{X_{i}^{2}+Y_{i}^{2}+Z_{i}^{2}}, \\
H_{i} & =\frac{1}{\text { Dist }_{i}} .
\end{aligned}
$$

(4) By substituting $H_{i}$ into the evaluation function of flavour concentration, the flavour concentration at the current position of the fruit fly is

$$
\text { Smell }_{i}=\text { function }\left(H_{i}\right) \text {, }
$$

and the individuals with the highest flavour concentration in the current fruit fly population are

$$
\text { [best Smell, best Index] }=\max \left(\operatorname{Smell}_{i}\right) \text {. }
$$

(5) To retain the best flavour concentration and the corresponding individual coordinates of the fruit fly population, the fruit fly populations use vision to locate food sources and then fly to the location of these food sources.

$$
\left\{\begin{array}{l}
\text { Smell best }=\text { best Smell, } \\
X_{0}=X(\text { best Index }), \\
Y_{0}=Y(\text { best Index }) \\
Z_{0}=Z \text { (best Index). }
\end{array}\right.
$$

(6) Iterative optimization is performed and repeated from Steps 2 to 5 to determine whether the best flavour concentration at present is better than that at the previous iteration, and mgen $<$ Mgen. If the previous inequality holds, the above Step 5 needs to be carried out.

For conducting parameter optimization based on the FOA, the population size is 30 , and the maximum number of iterations is 100 . For the four-axis horizontal boring machine tool, the position, and orientation affect the geometric accuracy together, especially when the geometric error and accuracy retentivity of each error vector are changed; therefore, the fitness functions should be considered together. With the geometric error model, the fitness function is developed with equation (42) as follows:

$$
\begin{aligned}
& f=\alpha\left\|\beta_{i}\right\|_{2}+\lambda\left\|\gamma_{a i}\right\|_{2}, \\
& \beta_{i}=\omega \sqrt{\frac{2 \sum_{p}^{n}\left(e_{p m}-e_{p i}\right)}{n\left(e_{p l}+e_{p u}\right)}},
\end{aligned}
$$

where $\beta_{i}$ is the root of the sum of squares of the fluctuating values of the geometric errors, which is defined as an influence factor for describing quantitatively the variation in the geometric error terms in service relative to those of the initial state. $e_{p l}$ and $e_{p u}$ are the upper and lower bounds of the allowance of the error terms, respectively. $\emptyset$ is constant for a particular geometric accuracy, and $e_{p l}$ and $e_{p u}$ are the measurement values and initial values of the geometric error terms, respectively. $\gamma_{a i}$ is the root of the sum of squares of the accuracy retentivity of the 6 position and orientation vectors.

According to the abovementioned analysis results, the order of magnitude of the influence factor in the components is $10^{1}$, and the order of magnitude of accuracy 


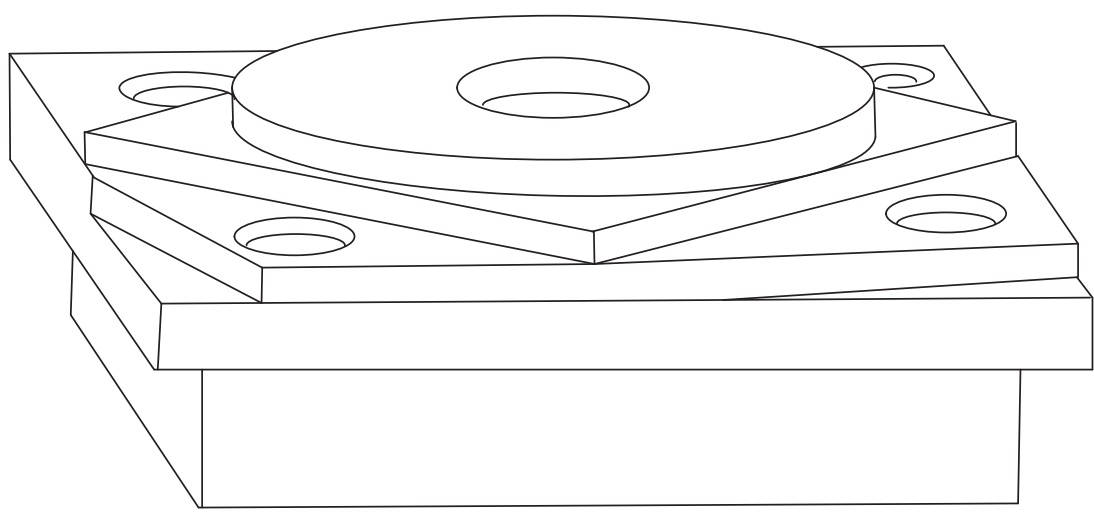

(a)

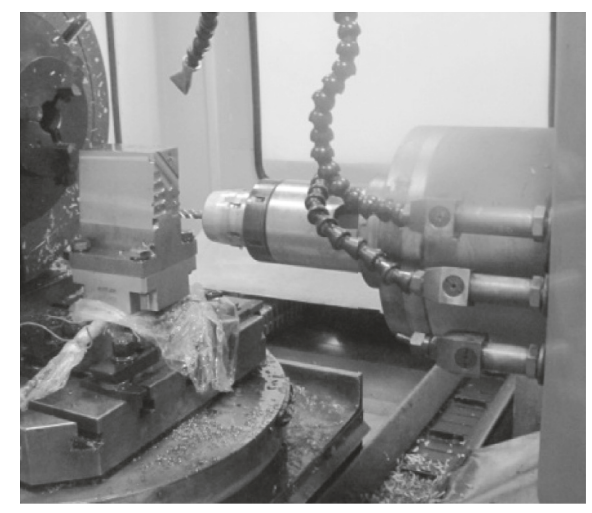

(b)

Figure 10: The 3D model and part machining. (a) The 3D model of the test piece. (b) Part machining on the four-axis machine tool.

retentivity is $10^{-1}$. The influence factor of geometric error is much larger than the value of accuracy retentivity, leading to lower-order functions that are easily missed in the initial optimization stage and a tendency to become trapped in local optimization and to diverge. Each function should be of the same order of magnitude to avoid the above scenario. When the relationship of the coefficients $\alpha$ and $\lambda$ is expressed in equation (44) as

$$
\alpha=\varsigma \lambda, \quad(\varsigma=100),
$$

the accuracy retentivity and influence factor can be of the same order of magnitude. The minor fluctuation in $\zeta$ does not affect the convergence speed or accuracy. The weight coefficient of the fitness function $\zeta$ is determined to simplify the calculation as $\alpha=1$ and $\lambda=10^{2}$.

Compensation values of each geometric error term in different locations can be obtained after the FOA is conducted, and the compensation values can be obtained. Standard test pieces selected are used for accuracy testing of the four-axis machine tool, as specified by ISO 10791-7 [51]. A comparison of the dimensional accuracy of two parts before and after compensation allows the effectiveness of the analysis results to be assessed. The test part is designed with Unigraphics software, as shown in Figure 10(a). The machining of the test part was conducted on the four-axis machine tool, as shown in Figure 10(b).

As shown in Figure 11, Part 1 was machined after the last geometric error measurement, and geometric error compensation was not implemented at this time. Part 2 was machined after 10 days with approximately 8 hours run per working day, and the geometric errors were compensated based on the FOA optimization results after Part 1 was machined. The same end milling cutter with a diameter of $32 \mathrm{~mm}$ was used to process all the outer surfaces of the specimens. The cutting parameters included a cutting speed of $40 \mathrm{~mm} / \mathrm{min}$, a feed per tooth of $0.05 \mathrm{~mm}$, and a radial cutting depth of $0.15 \mathrm{~mm}$. The parts were measured using a coordinate measuring machine after machining, and the precision index of the standard part is listed in Table 6.

According to the above comparison and analysis, it can be seen that the variation in precision on each item is lower

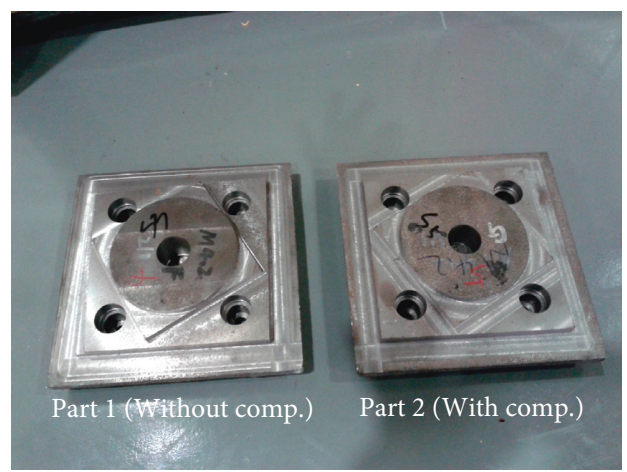

FIGURE 11: Parts machined without and with error compensation.

TABLE 6: Comparison of the measured results of the machined parts (mm).

\begin{tabular}{lcccc}
\hline \multirow{2}{*}{ Detection indexes } & \multirow{2}{*}{ Tolerance } & \multicolumn{2}{c}{ Test results } \\
& & & Part 1 & Part 2 \\
\hline \multirow{2}{*}{ Centre hole } & Cylindricity & 0.010 & 0.009 & 0.009 \\
\multirow{3}{*}{ Square } & Squareness & $\emptyset 0.010$ & 0.006 & 0.005 \\
& Straightness & 0.010 & 0.009 & 0.008 \\
Circle & Squareness & 0.013 & 0.010 & 0.010 \\
& Roundness & 0.016 & 0.014 & 0.016 \\
& Concentricity & $\varnothing 0.016$ & 0.008 & 0.006 \\
\hline
\end{tabular}

than $25.0 \%$, the average variation rate is $10.8 \%$, the maximum variance in the detection indexes between the machined parts is $0.002 \mathrm{~mm}$, and the precision of the size and form of the machined parts remains nearly constant. The machining accuracy of the machine tool is improved with the proposed geometric error modelling and compensation based on the analysis of geometric accuracy.

\section{Conclusions}

With the remarkable improvement in part design and processing accuracy, the role of accuracy retentivity in the performance of multiaxis machine tools has become increasingly prominent. The method of quantitative analysis and guaranteeing the retention capability of the geometric 
accuracy of multiaxis machine tools is an intractable problem. This paper proposes a recognition methodology for key geometric errors according to the analysis of accuracy retentivity of geometric errors. The conclusions of this research are as follows:

(1) Based on screw theory, a universal model is established to represent the kinematics of the four-axis machine tool without requiring establishing the local coordinate system and taking account of the relative location among machine tool components. The geometric accuracy of the machine tool is mapped to the time dimension and spatial scale, and an accuracy retentivity model is established for assessing the geometric accuracy in quasistatic service periods of multiaxis machine tools.

(2) A key geometric error identification method is proposed based on feature extraction; the correlation, similarity, and sensitivity of geometric error terms and error vectors are comprehensively considered. An assessment method of quantitative relationships based on feature extraction is designed, and then crucial geometric error terms are identified. Both the causality and the quantitative association are fully analysed. The results reveal that the effect of the PIGEs on the error vectors of the position and orientation is greater than that of the PDGEs of the linear and rotary axes.

(3) The accuracy retentivity is used to evaluate the performance of a multiaxis machine tool. The optimum values are obtained with the fruit fly algorithm, and the accuracy retentivity, which depends on geometric error, is considered and then used to determine NC instructions with mathematical expressions of the kinematic model. The measurement results of the machined parts show that the four-axis machine tool still maintains machine precision after geometric error compensation is conducted, in which the accuracy retentivity and influence factor caused by the variation in the geometric error are taken into account. The variation in precision on each item is lower than $25.0 \%$, and the maximum variance in the detection indexes between the machined parts is $0.002 \mathrm{~mm}$.

In this paper, we focus on modelling, recognition of key geometric errors, and compensation of geometric errors. However, thermal errors, cutting force-induced errors, and servo errors unavoidably affect the accuracy retentivity of the machine tool, which would cause the prediction models to gradually lose efficacy. The geometric accuracy monitoring method and machining test method need to be applied to evaluate accuracy retentivity in the longer term for further improvement of accuracy.

\section{Notation}

$$
\left[g_{b n}(0)\right]:
$$

The homogeneous transformation matrix of the $n$-th axis

\begin{tabular}{|c|c|}
\hline$\omega_{i}:$ & $\begin{array}{l}\text { Unit direction vector of rotary } \\
\text { axis, and }\end{array}$ \\
\hline$q_{i}:$ & Arbitrary points on the rotary axis \\
\hline Se (3): & $\begin{array}{l}\text { Set of } 3 \times 3 \text { matrices which satisfy } \\
\text { the special orthogonal with } \\
\text { respecting to unit direction vector } \\
\text { of rotary axis }\end{array}$ \\
\hline So (3): & $\begin{array}{l}\text { Set of } 3 \times 3 \text { matrices which satisfy } \\
\text { the special orthogonal with } \\
\text { respecting to arbitrary points on } \\
\text { the rotary axis }\end{array}$ \\
\hline $\mathbf{O}_{a}:$ & $\begin{array}{l}\text { Actual values of position error } \\
\text { vector }\end{array}$ \\
\hline $\mathbf{O}_{i}:$ & $\begin{array}{l}\text { Ideal values of position error } \\
\text { vector }\end{array}$ \\
\hline & 3-dimensional vector space \\
\hline$\omega_{0}=\left[\begin{array}{lll}w_{o x} & w_{o y} & w_{o z}\end{array}\right]^{1}:$ & $\begin{array}{l}\text { The offsets of workpiece } \\
\text { coordinate system relative to } \\
\text { reference coordinate system }\end{array}$ \\
\hline$t_{0}=\left[\begin{array}{lll}t_{o x} & t_{o y} & t_{o z}\end{array}\right]^{\mathrm{T}}:$ & $\begin{array}{l}\text { The offsets of tool coordinate } \\
\text { system relative to reference } \\
\text { coordinate system }\end{array}$ \\
\hline $\bar{P}_{k}:$ & $\begin{array}{l}\text { Mean values of position error } \\
\text { vector } k\end{array}$ \\
\hline $\bar{O}_{k}:$ & $\begin{array}{l}\text { Mean values of orientation error } \\
\text { vector } k\end{array}$ \\
\hline
\end{tabular}

\section{Data Availability}

The data used to support the findings of this study are included within the article.

\section{Conflicts of Interest}

The authors declare that there are no conflicts of interest regarding the publication of this paper.

\section{Acknowledgments}

This research was also supported by the Natural Science Foundation of Inner Mongolia (no. 2019BS05008) besides the Inner Mongolia University of Technology, Natural Science Foundation of China (no. RZ1900002164), the National Natural Science Foundation of China (no. 61763036), and the National Key R\&D Program of China (no. 2018YFB1307501).

\section{References}

[1] S. Aguado, D. Samper, J. Santolaria, and J. J. Aguilar, “Towards an effective identification strategy in volumetric error compensation of machine tools," Measurement Science and Technology, vol. 23, no. 6, pp. 1-12, 2012.

[2] L. Andolfatto, S. Lavernhe, and J. R. R. Mayer, "Evaluation of servo, geometric and dynamic error sources on five-axis highspeed machine tool," International Journal of Machine Tools and Manufacture, vol. 51, no. 10-11, pp. 787-796, 2011.

[3] M. Du, Z. Cheng, Y. Zhang, and S. Wang, "Multiobjective optimization of tool geometric parameters using genetic algorithm," Complexity, vol. 2018, pp. 1-14, 2018. 
[4] S. J. Guo, G. D. Jiang, D. S. Zhang, and X. S. Mei, "Positionindependent geometric error identification and global sensitivity analysis for the rotary axes of five-axis machine tools," Measurement Science and Technology, vol. 28, no. 4, pp. 1-14, 2017.

[5] R. Sato and K. Shirase, "Geometric error compensation of five-axis machining centers based on on-machine workpiece measurement," International Journal of Automation Technology, vol. 12, no. 2, pp. 230-237, 2018.

[6] S. Xiang, H. Li, M. Deng, and J. Yang, "Geometric error analysis and compensation for multi-axis spiral bevel gears milling machine," Mechanism and Machine Theory, vol. 121, pp. 59-74, 2018.

[7] R. Chhabra and M. R. Emami, "A generalized exponential formula for forward and differential kinematics of open-chain multi-body systems," Mechanism and Machine Theory, vol. 73, pp. 61-75, 2014.

[8] G. Q. Fu, J. Z. Fu, Y. T. Xu, and Z. C. Chen, "Product of exponential model for geometric error integration of multi-axis machine tools," The International Journal of Advanced Manufacturing Technology, vol. 71, no. 9-12, pp. 1653-1667, 2014.

[9] M. Givi and J. R. R. Mayer, "Optimized volumetric error compensation for five-axis machine tools considering relevance and compensability," CIRP Journal of Manufacturing Science and Technology, vol. 12, pp. 44-55, 2016.

[10] G.-C. Vosniakos and E. Matsas, "Improving feasibility of robotic milling through robot placement optimisation," Robotics and Computer-Integrated Manufacturing, vol. 26, no. 5, pp. 517-525, 2010.

[11] D. Li, P. Feng, J. Zhang, D. Yu, and Z. Wu, "An identification method for key geometric errors of machine tool based on matrix differential and experimental test," Proceedings of the Institution of Mechanical Engineers, Part C: Journal of Mechanical Engineering Science, vol. 228, no. 17, pp. 3141-3155, 2014.

[12] J. Yang, J. R. R. Mayer, and Y. Altintas, "A position independent geometric errors identification and correction method for five-axis serial machines based on screw theory," International Journal of Machine Tools and Manufacture, vol. 95, pp. 52-66, 2015.

[13] S. Xiang and Y. Altintas, "Modeling and compensation of volumetric errors for five-axis machine tools," International Journal of Machine Tools and Manufacture, vol. 101, pp. 6578, 2016.

[14] J.-D. Wang, Q.-J. Wang, and H.-T. Li, "The method of geometric error measurement of NC machine tool based on the principle of space vector's direction measurement," International Journal of Precision Engineering and Manufacturing, vol. 20, no. 4, pp. 511-524, 2019.

[15] G. Q. Fu, J. Z. Fu, H. Y. Shen, Y. T. Xu, and Y. A. Jin, "Productof-exponential formulas for precision enhancement of fiveaxis machine tools via geometric error modeling and compensation," The International Journal of Advanced Manufacturing Technology, vol. 81, no. 1-4, pp. 289-305, 2015.

[16] K. I. Lee, D. M. Lee, and S. H. Yang, "Parametric modeling and estimation of geometric errors for a rotary axis using double ball-bar," The International Journal of Advanced Manufacturing Technology, vol. 62, no. 5-8, pp. 741-750, 2012.

[17] Z. C. Du, J. Wu, and J. G. Yang, "Geometric error modeling and sensitivity analysis of single-axis assembly in three-axis vertical machine center based on jacobian-torsor model," ASCE-ASME Journal of Risk and Uncertainty in Engineering Systems, Part B: Mechanical Engineering, vol. 4, no. 3, pp. 1-12, 2018.

[18] H. Tang, J.-A. Duan, and S. Lu, "Stream-of-variation (SOV) theory applied in geometric error modeling for six-axis motion platform," IEEE Transactions on Systems, Man, and Cybernetics: Systems, vol. 99, pp. 1-9, 2017.

[19] J. W. Fan, H. Tao, C. Wu, R. Pan, Y. Tang, and Z. Li, "Kinematic errors prediction for multi-axis machine tools' guideways based on tolerance," The International Journal of Advanced Manufacturing Technology, vol. 98, no. 5-8, pp. 1131-1144, 2018.

[20] A. Los and J. R. R. Mayer, "Application of the adaptive Monte Carlo method in a five-axis machine tool calibration uncertainty estimation including the thermal behavior," Precision Engineering, vol. 53, pp. 17-25, 2018.

[21] L. Cai, Z. Zhang, Q. Cheng, Z. Liu, P. Gu, and Y. Qi, “An approach to optimize the machining accuracy retainability of multi-axis NC machine tool based on robust design," Precision Engineering, vol. 43, pp. 370-386, 2016.

[22] S. Ibaraki, I. Yoshida, and T. Asano, "A machining test to identify rotary axis geometric errors on a five-axis machine tool with a swiveling rotary table for turning operations," Precision Engineering, vol. 55, pp. 22-32, 2019.

[23] J. Li, F. G. Xie, and X. J. Liu, "Geometric error modeling and sensitivity analysis of a five-axis machine tool," vol. 82, no. 9-12, pp. 2037-2051, 2016.

[24] P. A. Vanrolleghem, G. Mannina, A. Cosenza, and M. B. Neumann, "Global sensitivity analysis for urban water quality modelling: terminology, convergence and comparison of different methods," Journal of Hydrology, vol. 522, pp. 339-352, 2015.

[25] S. Moro, P. Rita, and B. Vala, "Predicting social media performance metrics and evaluation of the impact on brand building: a data mining approach," Journal of Business Research, vol. 69, no. 9, pp. 3341-3351, 2016.

[26] G. Chandrashekar and F. Sahin, "A survey on feature selection methods," Computers \& Electrical Engineering, vol. 40, no. 1, pp. 16-28, 2014.

[27] N. Barraza, S. Moro, M. Ferreyra, and A. de la Peña, "Mutual information and sensitivity analysis for feature selection in customer targeting: a comparative study," Journal of Information Science, vol. 45, no. 1, pp. 53-67, 2019.

[28] P. Cortez and M. J. Embrechts, "Using sensitivity analysis and visualization techniques to open black box data mining models," Information Sciences, vol. 225, pp. 1-17, 2013.

[29] Y. Q. Wang, J. K. Wu, H. B. Liu, K. Kang, and K. Liu, "Geometric accuracy long-term continuous monitoring using strain gauges for CNC machine tools," The International Journal of Advanced Manufacturing Technology, vol. 98, no. 5-8, pp. 1145-1153, 2018.

[30] J. X. Ma, W. H. Zhao, and G. B. Zhang, "Research status and analyses on accuracy retentivity of do-mestic CNC machine tools," China Mechanical Engineering, vol. 26, no. 22, pp. 3108-3115, 2015.

[31] Y. Tan, L. Zhang, and Y. Hu, "A wear model of plane sliding pairs based on fatigue contact analysis of asperities," Tribology Transactions, vol. 58, no. 1, pp. 148-157, 2015.

[32] J. Y. Guo, Y. F. Li, B. Zheng, and H. Z. Huang, "Bayesian degradation assessment of CNC machine tools considering unit non-homogeneity," Journal of Manufacturing Science and Technology, vol. 32, no. 6, pp. 2479-2485, 2016.

[33] H. Liu, J. Wu, K. Liu et al., "Pretightening sequence planning of anchor bolts based on structure uniform deformation for large CNC machine tools," International Journal of Machine Tools and Manufacture, vol. 136, pp. 1-18, 2019.

[34] K.-C. Fan, H.-M. Chen, and T.-H. Kuo, "Prediction of machining accuracy degradation of machine tools," Precision Engineering, vol. 36, no. 2, pp. 288-298, 2012. 
[35] V. Portman, I. Inasaki, M. Sakakura, and M. Iwatate, "Formshaping systems of machine tools: theory and applications," CIRP Annals, vol. 47, no. 1, pp. 329-332, 1998.

[36] ISO 230-1, Test Code for Machine Tools-Part 1: Geometric Accuracy of Machines Operating under No-Load or Quasticstatic Conditions, ISO, Geneva, Switzerland, 2012.

[37] Q. Cheng, C. Wu, P. H. Gu, W. F. Chang, and D. S. Xuan, "An analysis methodology for stochastic characteristic of volumetric error in multiaxis CNC machine tool," Mathematical Problems in Engineering, vol. 2013, Article ID 863283, 12 pages, 2013.

[38] S. J. Guo, G. D. Jiang, and X. S. Mei, "Investigation of sensitivity analysis and compensation parameter optimization of geometric error for five-axis machine tool," The International Journal of Advanced Manufacturing Technology, vol. 93, no. 9-12, pp. 3229-3243, 2017.

[39] S. J. Guo, X. S. Mei, and G. D. Jiang, "Geometric accuracy enhancement of five-axis machine tool based on error analysis," The International Journal of Advanced Manufacturing Technology, 2019.

[40] J. Guo, B. Li, Z. Liu, J. Hong, and Q. Zhou, "A new solution to the measurement process planning for machine tool assembly based on Kalman filter," Precision Engineering, vol. 43, pp. 356-369, 2016.

[41] R. M. Murray, A Mathematical Introduction to Robotic Manipulation, CRC Press, Boca Raton, FL, USA, 1994.

[42] C. Hong, S. Ibaraki, and A. Matsubara, "Influence of positiondependent geometric errors of rotary axes on a machining test of cone frustum by five-axis machine tools," Precision Engineering, vol. 35, no. 1, pp. 1-11, 2011.

[43] G.-J. Park, T.-H. Lee, K. H. Lee, and K.-H. Hwang, "Robust design: an overview," AIAA Journal, vol. 44, no. 1, pp. 181-191, 2006.

[44] K. Kim and M. K. Kim, "Volumetric accuracy analysis based on generalized geometric error model in multi-axis machine tools," Mechanism and Machine Theory, vol. 26, no. 2, pp. 207-219, 1991.

[45] B. Iooss and A. Saltelli, "Introduction to sensitivity analysis," in Handbook of Uncertainty Quantification, Springer International Publishing, Switzerland, 2017.

[46] J. Wang and J. Zhang, "Big data analytics for forecasting cycle time in semiconductor wafer fabrication system," International Journal of Production Research, vol. 54, no. 23, pp. 7231-7244, 2016.

[47] A. Wosiak and D. Zakrzewska, "Integrating correlation-based feature selection and clustering for improved cardiovascular disease diagnosis," Complexity, vol. 2018, Article ID 2520706, 11 pages, 2018.

[48] K. Cheng, Z. Lu, and K. Zhang, "Multivariate output global sensitivity analysis using multi-output support vector regression," Structural and Multidisciplinary Optimization, vol. 59, no. 6, pp. 2177-2187, 2019.

[49] L. Jiang, G. Ding, Z. Li, S. Zhu, and S. Qin, "Geometric error model and measuring method based on worktable for fiveaxis machine tools," Proceedings of the Institution of $\mathrm{Me}$ chanical Engineers, Part B: Journal of Engineering Manufacture, vol. 227, no. 1, pp. 32-44, 2013.

[50] S. Kanarachos, J. Griffin, and M. E. Fitzpatrick, "Efficient truss optimization using the contrast-based fruit fly optimization algorithm," Computers \& Structures, vol. 182, pp. 137-148, 2017.

[51] ISO 10791-7, Test Conditions for Machining Centres-Part 7: Accuracy of Finished Test Piece, ISO, Geneva, Switzerland, 2014. 


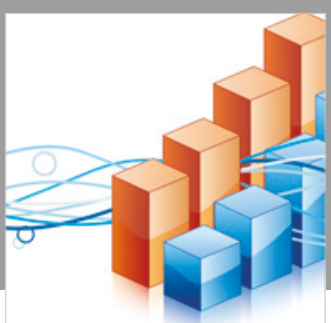

Advances in

Operations Research

\section{-n-m}
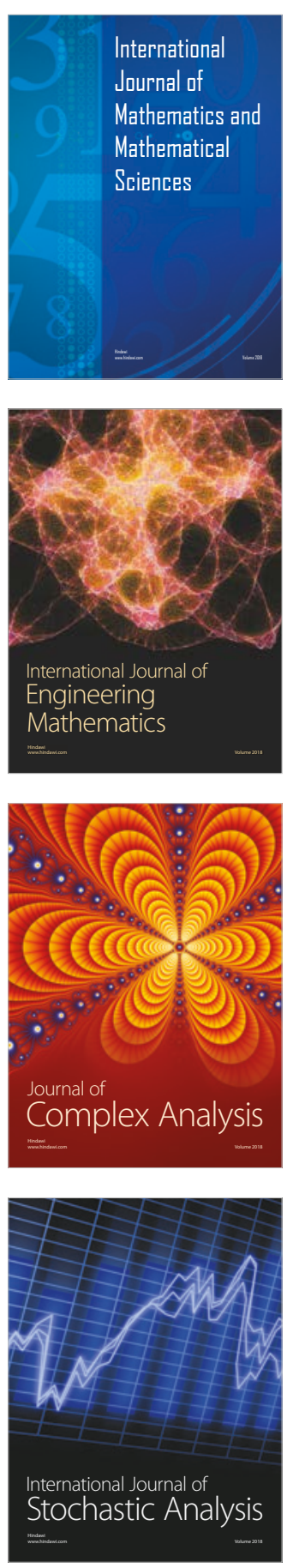
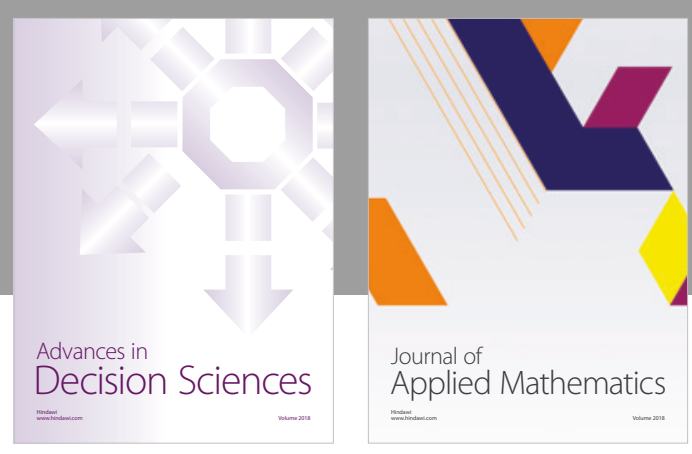

Journal of

Applied Mathematics
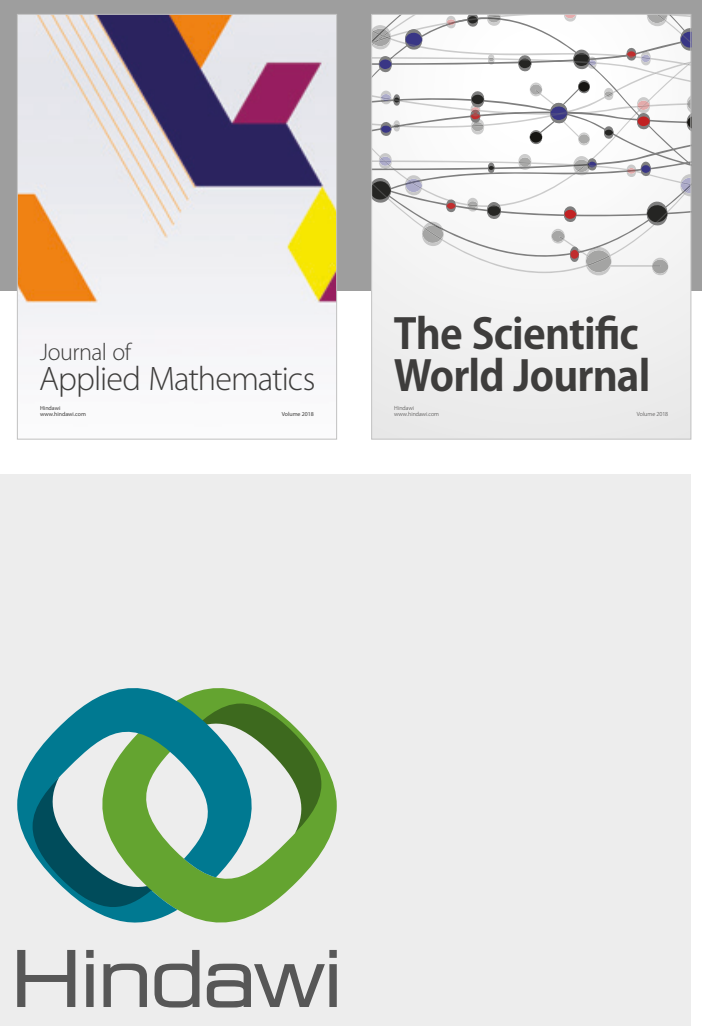

Submit your manuscripts at

www.hindawi.com

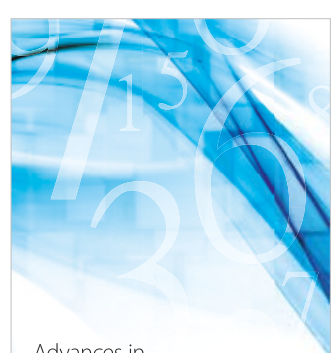

Advances in
Numerical Analysis
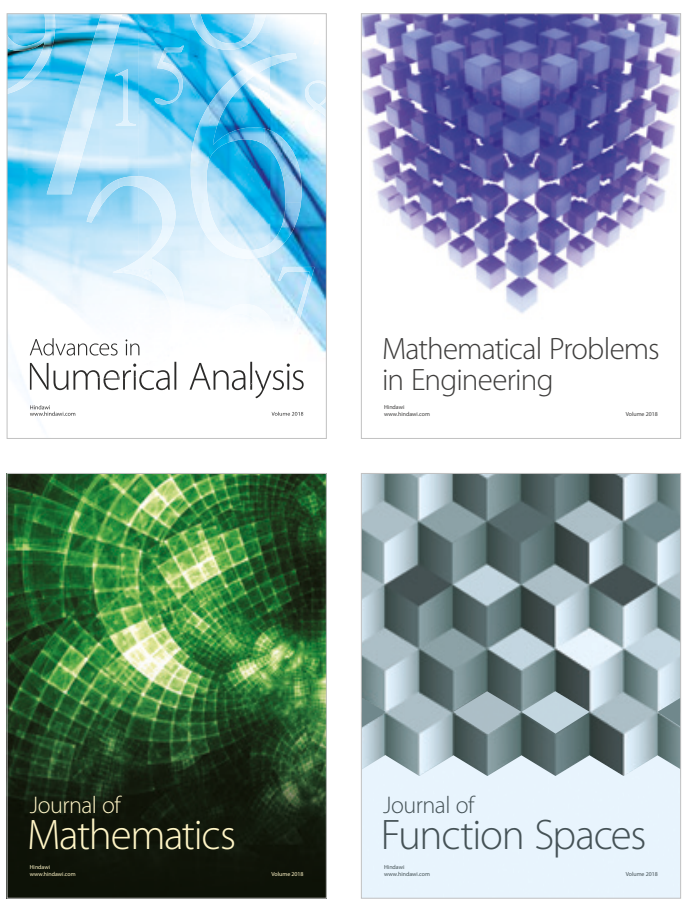

Mathematical Problems in Engineering

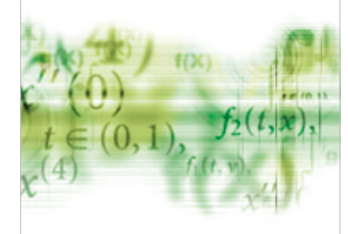

International Journal of

Differential Equations

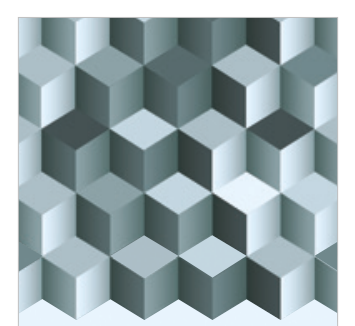

Journal of

Function Spaces
The Scientific

World Journal

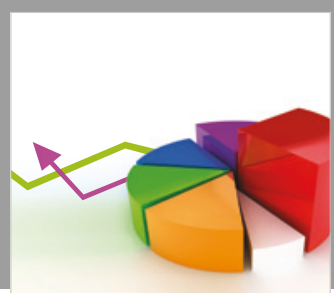

Journal of

Probability and Statistics
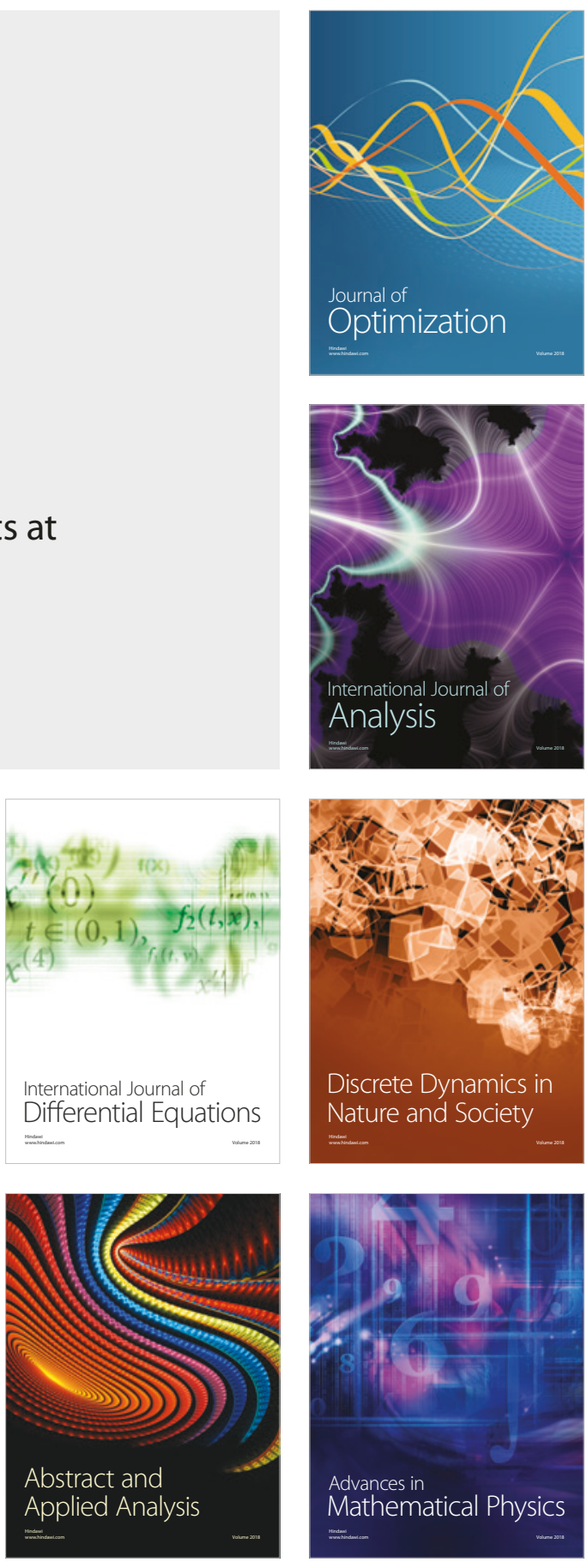